\title{
FINITENESS THEOREMS FOR COMMUTING AND SEMICONJUGATE RATIONAL FUNCTIONS
}

\author{
FEDOR PAKOVICH
}

\begin{abstract}
Let $B$ be a fixed rational function of one complex variable of degree at least two. In this paper, we study solutions of the functional equation $A \circ X=X \circ B$ in rational functions $A$ and $X$. Our main result states that, unless $B$ is a Lattès map or is conjugate to $z^{ \pm d}$ or $\pm T_{d}$, the set of solutions is finite, up to some natural transformations. In more detail, we show that there exist finitely many rational functions $A_{1}, A_{2}, \ldots, A_{r}$ and $X_{1}, X_{2}, \ldots, X_{r}$ such that the equality $A \circ X=X \circ B$ holds if and only if there exists a Möbius transformation $\mu$ such that $A=\mu \circ A_{j} \circ \mu^{-1}$ and $X=\mu \circ X_{j} \circ B^{\circ k}$ for some $j, 1 \leq j \leq r$, and $k \geq 1$. We also show that the number $r$ and the degrees $\operatorname{deg} X_{j}, 1 \leq j \leq r$, can be bounded from above in terms of the degree of $B$ only. As an application, we prove an effective version of the classical theorem of Ritt about commuting rational functions.
\end{abstract}

\section{INTRODUCTION}

Commuting rational functions of one complex variable, that is, rational solutions of the functional equation

$$
B \circ X=X \circ B
$$

were investigated already at the dawn of complex dynamics in the papers of Fatou, Julia, and Ritt [6], [8, 23]. The most general result was obtained by Ritt. Roughly speaking, it states that solutions of equation (10) having no iterate in common reduce either to powers, or to Chebyshev polynomials, or to Lattès maps. More precisely, in its modern formulation due to Eremenko ([3]), the Ritt theorem asserts that if $X$ and $B$ are commuting rational functions of degree at least two having no iterate in common, then there exists an orbifold $\mathcal{O}$ of zero Euler characteristic defined on $\mathbb{C P}^{1}, \mathbb{C}$, or $\mathbb{C}^{*}$ such that $A: \mathcal{O} \rightarrow \mathcal{O}$ and $B: \mathcal{O} \rightarrow \mathcal{O}$ are covering maps between orbifolds. Notice that the Ritt theorem provides no information about commuting rational functions such that

$$
X^{\circ l}=B^{\circ k}
$$

for some $l, k \geq 1$, and a characterization of pairs $X$ and $B$ (commuting or not) satisfying (22) is known only in the polynomial case (22], 23]). Simple examples of commuting rational functions $X$ and $B$ satisfying (2) can be obtained setting

$$
X=\mu_{1} \circ R^{\circ l_{1}}, \quad B=\mu_{2} \circ R^{\circ l_{2}},
$$

Received by the editors April 23, 2019, and, in revised form, July 11, 2020.

2010 Mathematics Subject Classification. Primary 30D05, 37P05.

This research was partially supported by the ISF, Grants No. 1432/18. 
where $R$ is an arbitrary rational function and $\mu_{1}, \mu_{2}$ are Möbius transformations commuting with $R$ and between themselves. However, it was shown already by Ritt ([23]) that other examples also exist.

Functional equation (11) is a particular case of the functional equation

$$
A \circ X=X \circ B
$$

where $A, B$, and $X$ are rational functions, playing along with equation (1) an important role in complex and arithmetic dynamics (see e.g. [1, 3], 4], 7], 11, [17], [21]). We always will assume that $A$ and $B$ in (3) have degree at least two, while $X$ has degree at least one. In case if (3) is satisfied for some $X$ with $\operatorname{deg} X \geq 2$, the function $B$ is called semiconjugate to the function $A$, and the function $X$ is called a semiconjugacy from $B$ to $A$. The case $\operatorname{deg} X=1$ corresponds to the usual conjugacy. In terms of dynamical systems, the conjugacy condition means that the dynamical systems $B^{\circ k}, k \geq 1$, and $A^{\circ k}, k \geq 1$, are equivalent, while the semiconjugacy condition means that the system $A^{\circ k}, k \geq 1$, is a factor of the system $B^{\circ k}, k \geq 1$.

Semiconjugate rational functions were investigated at length in the recent papers [14], 19, 20]. In particular, it was shown in [14 that if a solution $A, B, X$ of equation (3) is primitive, that is, satisfying the condition $\mathbb{C}(X, B)=\mathbb{C}(z)$, then there exist orbifolds $\mathcal{O}_{1}$ and $\mathcal{O}_{2}$ of non-negative Euler characteristic defined on $\mathbb{C P}^{1}$ such that $A: \mathcal{O}_{1} \rightarrow \mathcal{O}_{1}, B: \mathcal{O}_{2} \rightarrow \mathcal{O}_{2}$, and $X: \mathcal{O}_{1} \rightarrow \mathcal{O}_{2}$ are minimal holomorphic maps between orbifolds. This condition generalizes the condition provided by the Ritt theorem, and implies strong restrictions on the possible form of $A, B$ and $X$. In particular, it implies that the Galois closure of the field extension $\mathbb{C}(z) / \mathbb{C}(X)$ has genus zero or one.

Any solution of (3) reduces to a primitive one by a simple iterative process. Indeed, if $\mathbb{C}(X, B) \neq \mathbb{C}(z)$, then by the Lüroth theorem $\mathbb{C}(X, B)=\mathbb{C}\left(U_{1}\right)$ for some rational function $U_{1}$ of degree greater than one, and hence

$$
X=X_{1} \circ U_{1}, \quad B=V_{1} \circ U_{1}
$$

for some rational functions $X_{1}$ and $V_{1}$. Substituting these expressions in (3) we see that the triple $A, X_{1}, U_{1} \circ V_{1}$ is another solution of (3). This new solution is not necessary primitive. Nevertheless, $\operatorname{deg} X_{1}<\operatorname{deg} X$. Therefore, after a finite number of similar transformations we will arrive at a primitive solution.

In this paper, we study the totality of solutions of (3) in rational functions $A$ and $X$ for a fixed rational function $B$. We show that, unless $B$ has a very special form, the number of solutions of (3), considered up to some natural transformations, is finite. Moreover, this number can be bounded from above in terms of the degree of $B$ only. Notice that the results of [14, 19, [20, do not immediately imply any results of this kind, since a priori the number of steps in the reduction to a primitive solution as well as the number of primitive solutions for a fixed function $B$ can be arbitrarily large.

In more details, let $A, X$ be a solution of (3). It is clear that $A$ is defined by $X$ in a unique way. However, for a given rational function $A$ there might be several $X$ satisfying (3). In particular, for any $k \geq 1$ we may replace $X$ by $\widetilde{X}=X \circ B^{\circ k}$. More generally, if $A, X$ is a solution of (3), then for any Möbius transformation $\mu$ and $k \geq 1$ we obtain another solution setting

$$
\widetilde{A}=\mu \circ A \circ \mu^{-1}, \quad \widetilde{X}=\mu \circ X \circ B^{\circ k} .
$$


We say that a rational function $B$ is special if it is either a Lattès map, or it is conjugate to $z^{ \pm n}$ or $\pm T_{n}$. In these terms, the main result of the paper is following.

Theorem 1.1. Let $B$ be a non-special rational function of degree at least two. Then there exist rational functions $A_{1}, A_{2}, \ldots, A_{r}$ and $X_{1}, X_{2}, \ldots, X_{r}$ such that rational functions $A$ and $X$ satisfy (3) if and only if

$$
A=\mu \circ A_{j} \circ \mu^{-1}, \quad X=\mu \circ X_{j} \circ B^{\circ k}
$$

for some $j, 1 \leq j \leq r$, Möbius transformation $\mu$, and $k \geq 0$. Furthermore, there exist (computable) functions $\psi, \varphi: \mathbb{N} \rightarrow \mathbb{N}$ such that for any non-special $B$ of degree $d$ the number $r$ and the degrees $\operatorname{deg} X_{j}, 1 \leq j \leq r$, are bounded from above by $\psi(d)$ and $\varphi(d)$, correspondingly.

Notice that Theorem 1.1 bounds both the number of conjugacy classes of rational functions $A$ such that (3) holds for some $X$, and, up to the second transformation in (4), the number of rational functions $X$ such that (3) holds for a fixed rational function $A$. In particular, for equation (11) considered as a particular case of equation (3) Theorem 1.1 implies the following result.

Theorem 1.2. Let $B$ be a non-special rational function of degree at least two. Then there exist rational functions $X_{1}, X_{2}, \ldots, X_{s}$ commuting with $B$ such that $a$ rational function $X$ commutes with $B$ if and only if

$$
X=X_{j} \circ B^{\circ k}
$$

for some $j, 1 \leq j \leq s$, and $k \geq 0$. Furthermore, there exist (computable) functions $\delta, \nu: \mathbb{N} \rightarrow \mathbb{N}$ such that for any non-special $B$ of degree $d$ the number $s$ and the degrees $\operatorname{deg} X_{j}, 1 \leq j \leq r$, are bounded from above by $\delta(d)$ and $\nu(d)$, correspondingly.

Notice that Theorem 1.2 immediately implies the Ritt theorem about commuting rational functions in its part concerning non-special functions. Indeed, if $X$ commutes with $B$, then any iterate $X^{\circ}$ does. Thus, it follows from Theorem 1.2 by the Dirichlet box principle that there exist distinct $l_{1}, l_{2} \leq \delta(d)+1$ such that

$$
X^{\circ l_{1}}=X_{j} \circ B^{\circ k_{1}}, \quad X^{\circ l_{2}}=X_{j} \circ B^{\circ k_{2}}
$$

for the same $j$ and some $k_{1}, k_{2} \geq 0$. Assuming that $l_{2}>l_{1}$, this yields

$$
X^{\circ l_{2}}=X^{\circ l_{1}} \circ B^{\circ\left(k_{2}-k_{1}\right)},
$$

implying that equality (2) holds for $l=l_{2}-l_{1}$ and $k=k_{2}-k_{1}$, since $X$ and $B$ commute. Moreover, $l$ satisfies the inequality $l \leq \delta(d)$. Thus, Theorem 1.2 improves the Ritt theorem, which provides neither existence of finitely many functions such that any function $X$ commuting with $B$ has form (5), nor boundedness of $l$ in terms of $\operatorname{deg} B$.

The paper is organized as follows. In the second section, we fix the notation and recall main definitions and results related to Riemann surface orbifolds. We also collect some technical results, mostly from the papers [14, [20], used in the following. In the third section, we study the systems of functional equations

$$
U_{i} \circ V_{i}=V_{i+1} \circ U_{i+1}, \quad 1 \leq i \leq s-1,
$$

where $U_{i}, V_{i}, 1 \leq i \leq s$, are rational functions of degree at least two, which we will call chains. Such chains correspond to chains

$$
B \rightarrow B_{1} \rightarrow B_{2} \rightarrow \cdots \rightarrow B_{s}
$$


of rational functions

$$
B=V_{1} \circ U_{1}, \quad B_{i}=U_{i} \circ V_{i}, \quad 1 \leq i \leq s,
$$

relating an arbitrary solution of (3) with a primitive one, and the main result of the third section asserts that under certain restrictions the length $s$ of such a chain can be effectively bounded in terms of the degree of $B$.

In the fourth section, we define an extended symmetry group of a rational function $F$ as the group of Möbius transformations $\sigma$ such that

$$
F \circ \sigma=\nu \circ F
$$

for some Möbius transformations $\nu$. We show that, unless $F=\mu_{1} \circ z^{n} \circ \mu_{2}$ for some Möbius transformations $\mu_{1}, \mu_{2}$, this group is finite, and using this fact prove Theorem 1.1 for primitive solutions of (3). In the fifth section, we prove a "compositional" counterpart of Theorem 1.1, which asserts that if $B$ is non-special, then any $X$ such that (3) holds for some $A$ can be decomposed into a composition of rational functions

$$
X=X^{\prime} \circ U \circ B^{\circ k}, \quad k \geq 1,
$$

such that the Galois closure of $\mathbb{C}(z) / \mathbb{C}\left(X^{\prime}\right)$ has genus zero or one, and $U$ is a "compositional right factor" of some iterate $B^{\circ l}$ with $l$ bounded in terms of $\operatorname{deg} B$. We also prove Theorem 1.1 and Theorem 1.2. Finally, we prove an effective version of the Ritt theorem.

\section{FunCtional DeCOMPOSITIONS AND ORBIFOLDS}

2.1. Orbifolds and maps between orbifolds. In this section we fix the notation and recall main definitions and results related to Riemann surface orbifolds (see 13, Appendix E). We also collect some technical results, from the papers [14, [15], 18], [20, used in the following.

A pair $\mathcal{O}=(R, \nu)$ consisting of a Riemann surface $R$ and a ramification function $\nu: R \rightarrow \mathbb{N}$ which takes the value $\nu(z)=1$ except at isolated points is called an orbifold. For an orbifold $\mathcal{O}$ the Euler characteristic of $\mathcal{O}$ is the number

$$
\chi(\mathcal{O})=\chi(R)+\sum_{z \in \mathbb{C P}^{1}}\left(\frac{1}{\nu(z)}-1\right),
$$

the set of singular points of $\mathcal{O}$ is the set

$$
c(\mathcal{O})=\left\{z_{1}, z_{2}, \ldots, z_{s}, \ldots\right\}=\left\{z \in \mathbb{C P}^{1} \mid \nu(z)>1\right\},
$$

and the signature of $\mathcal{O}$ is the set

$$
\nu(\mathcal{O})=\left\{\nu\left(z_{1}\right), \nu\left(z_{2}\right), \ldots, \nu\left(z_{s}\right), \ldots\right\} .
$$

If $R_{1}, R_{2}$ are Riemann surfaces provided with ramification functions $\nu_{1}, \nu_{2}$, and $f: R_{1} \rightarrow R_{2}$ is a holomorphic branched covering map, then $f$ is called a covering map $f: \mathcal{O}_{1} \rightarrow \mathcal{O}_{2}$ between orbifolds $\mathcal{O}_{1}=\left(R_{1}, \nu_{1}\right)$ and $\mathcal{O}_{2}=\left(R_{2}, \nu_{2}\right)$ if for any $z \in R_{1}$ the equality

$$
\nu_{2}(f(z))=\nu_{1}(z) \operatorname{deg}_{z} f
$$

holds, where $\operatorname{deg}_{z} f$ stands for the local degree of $f$ at the point $z$. If for any $z \in R_{1}$ instead of equality (7) the weaker condition

$$
\nu_{2}(f(z)) \mid \nu_{1}(z) \operatorname{deg}_{z} f
$$


holds, then the map $f$ is called a holomorphic map $f: \mathcal{O}_{1} \rightarrow \mathcal{O}_{2}$ between orbifolds $\mathcal{O}_{1}=\left(R_{1}, \nu_{1}\right)$ and $\mathcal{O}_{2}=\left(R_{2}, \nu_{2}\right)$.

A universal covering of an orbifold $\mathcal{O}=(R, \nu)$ is a covering map between orbifolds $\theta_{\mathcal{O}}: \widetilde{\mathcal{O}} \rightarrow \mathcal{O}$ such that $\widetilde{R}$ is simply connected and $\widetilde{\nu}(z) \equiv 1$. If $\theta_{\mathcal{O}}$ is such a map, then there exists a group $\Gamma_{\mathcal{O}}$ of conformal automorphisms of $\widetilde{R}$ such that the equality $\theta_{\mathcal{O}}\left(z_{1}\right)=\theta_{\mathcal{O}}\left(z_{2}\right)$ holds for $z_{1}, z_{2} \in \widetilde{R}$ if and only if $z_{1}=\sigma\left(z_{2}\right)$ for some $\sigma \in \Gamma_{\mathcal{O}}$. A universal covering exists and is unique up to a conformal isomorphism of $\widetilde{R}$, unless $\mathcal{O}$ is the Riemann sphere with one ramified point, or the Riemann sphere with two ramified points $z_{1}, z_{2}$ such that $\nu\left(z_{1}\right) \neq \nu\left(z_{2}\right)$ (see [5], Section IV.9.12). Abusing notation we will denote by $\widetilde{\mathcal{O}}$ both the orbifold and the Riemann surface $\widetilde{R}$.

Covering maps between orbifolds lift to isomorphisms between their universal coverings. More generally, for holomorphic maps between orbifolds the following proposition holds (see [14, Proposition 3.1]).

Proposition 2.1. Let $f: \mathcal{O}_{1} \rightarrow \mathcal{O}_{2}$ be a holomorphic map between orbifolds. Then for any choice of $\theta_{\mathcal{O}_{1}}$ and $\theta_{\mathcal{O}_{2}}$ there exist a holomorphic map $F: \widetilde{\mathcal{O}_{1}} \rightarrow \widetilde{\mathcal{O}_{2}}$ and a homomorphism $\varphi: \Gamma_{\mathcal{O}_{1}} \rightarrow \Gamma_{\mathcal{O}_{2}}$ such that the diagram

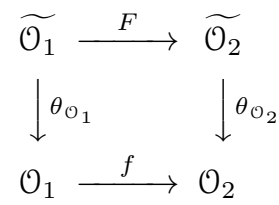

is commutative and for any $\sigma \in \Gamma_{\mathcal{O}_{1}}$ the equality

$$
F \circ \sigma=\varphi(\sigma) \circ F
$$

holds. The map $F$ is defined by $\theta_{\mathrm{O}_{1}}, \theta_{\mathrm{O}_{2}}$, and $f$ uniquely up to a transformation $F \rightarrow g \circ F$, where $g \in \Gamma_{\mathcal{O}_{2}}$. In the other direction, for any holomorphic map $F: \widetilde{\mathcal{O}_{1}} \rightarrow \widetilde{\mathcal{O}_{2}}$ which satisfies (10) for some homomorphism $\varphi: \Gamma_{\mathcal{O}_{1}} \rightarrow \Gamma_{\mathcal{O}_{2}}$ there exists a uniquely defined holomorphic map between orbifolds $f: \mathcal{O}_{1} \rightarrow \mathcal{O}_{2}$ such that diagram (9) is commutative. The holomorphic map $F$ is an isomorphism if and only if $f$ is a covering map between orbifolds.

If $f: \mathcal{O}_{1} \rightarrow \mathcal{O}_{2}$ is a covering map between orbifolds $\mathcal{O}_{1}$ and $\mathcal{O}_{2}$ with compact supports $R_{1}$ and $R_{2}$, then the Riemann-Hurwitz formula implies that

$$
\chi\left(\mathcal{O}_{1}\right)=d \chi\left(\mathcal{O}_{2}\right),
$$

where $d=\operatorname{deg} f$ (see [13]). For holomorphic maps the following statement is true (see [14, Proposition 3.2]).

Proposition 2.2. Let $f: \mathcal{O}_{1} \rightarrow \mathcal{O}_{2}$ be a holomorphic map between orbifolds with compact supports. Then

$$
\chi\left(\mathcal{O}_{1}\right) \leq \chi\left(\mathcal{O}_{2}\right) \operatorname{deg} f,
$$

and the equality holds if and only if $f: \mathcal{O}_{1} \rightarrow \mathcal{O}_{2}$ is a covering map between orbifolds.

Let $R_{1}, R_{2}$ be Riemann surfaces and $f: R_{1} \rightarrow R_{2}$ a holomorphic branched covering map. Assume that $R_{2}$ is provided with ramification function $\nu_{2}$. In order to define a ramification function $\nu_{1}$ on $R_{1}$ so that $f$ would be a holomorphic map between orbifolds $\mathcal{O}_{1}=\left(R_{1}, \nu_{1}\right)$ and $\mathcal{O}_{2}=\left(R_{2}, \nu_{2}\right)$ we must satisfy condition (8), 
and it is easy to see that for any $z \in R_{1}$ a minimum possible value for $\nu_{1}(z)$ is defined by the equality

$$
\nu_{2}(f(z))=\nu_{1}(z) \operatorname{GCD}\left(\operatorname{deg}_{z} f, \nu_{2}(f(z)) .\right.
$$

In case if (13) is satisfied for any $z \in R_{1}$ we say that $f$ is a minimal holomorphic map between orbifolds $\mathcal{O}_{1}=\left(R_{1}, \nu_{1}\right)$ and $\mathcal{O}_{2}=\left(R_{2}, \nu_{2}\right)$.

It follows from the definition that for any orbifold $\mathcal{O}=(R, \nu)$ and a holomorphic branched covering map $f: R^{\prime} \rightarrow R$ there exists a unique orbifold structure $\mathcal{O}^{\prime}=\left(R^{\prime}, \nu^{\prime}\right)$ such that $f: \mathcal{O}^{\prime} \rightarrow \mathcal{O}$ is a minimal holomorphic map between orbifolds. We will denote the corresponding orbifold by $f^{*} \mathcal{O}$. Notice that any covering map between orbifolds $f: \mathcal{O}_{1} \rightarrow \mathcal{O}_{2}$ is a minimal holomorphic map. In particular, this implies that for any covering map $f: \mathcal{O}_{1} \rightarrow \mathcal{O}_{2}$ the equality $\mathcal{O}_{1}=f^{*} \mathcal{O}_{2}$ holds. Minimal holomorphic maps between orbifolds possess the following fundamental property with respect to the operation of composition (see [14, Theorem 4.1]).

Theorem 2.3. Let $f: R^{\prime \prime} \rightarrow R^{\prime}$ and $g: R^{\prime} \rightarrow R$ be holomorphic branched covering maps, and $\mathcal{O}=(R, \nu)$ an orbifold. Then

$$
(g \circ f)^{*} \mathcal{O}=f^{*}\left(g^{*} \mathcal{O}\right) .
$$

Theorem 2.3 implies in particular the following corollaries (see [14, Corollary 4.1 and Corollary 4.2]).

Corollary 2.4. Let $f: \mathcal{O}_{1} \rightarrow \mathcal{O}^{\prime}$ and $g: \mathcal{O}^{\prime} \rightarrow \mathcal{O}_{2}$ be minimal holomorphic maps (resp. covering maps) between orbifolds. Then $g \circ f: \mathcal{O}_{1} \rightarrow \mathcal{O}_{2}$ is a minimal holomorphic map (resp. covering map).

Corollary 2.5. Let $f: R_{1} \rightarrow R^{\prime}$ and $g: R^{\prime} \rightarrow R_{2}$ be holomorphic branched covering maps, and $\mathcal{O}_{1}=\left(R_{1}, \nu_{1}\right)$ and $\mathcal{O}_{2}=\left(R_{2}, \nu_{2}\right)$ orbifolds. Assume that $g \circ f: \mathcal{O}_{1} \rightarrow \mathcal{O}_{2}$ is a minimal holomorphic map (resp. a covering map). Then $g: g^{*} \mathrm{O}_{2} \rightarrow \mathrm{O}_{2}$ and $f: \mathcal{O}_{1} \rightarrow g^{*} \mathcal{O}_{2}$ are minimal holomorphic maps (resp. covering maps).

In this paper, essentially all considered orbifolds will be defined on $\mathbb{C P}^{1}$. So, we will omit the Riemann surface $R$ in the definition of $\mathcal{O}=(R, \nu)$ meaning that $R=\mathbb{C P}^{1}$. "Most" orbifolds on $\mathbb{C P}^{1}$ have negative Euler characteristic. Orbifolds $\mathcal{O}$ with $\chi(\mathcal{O}) \geq 0$ and corresponding $\Gamma_{\mathcal{O}}$ and $\theta_{\mathcal{O}}$ can be described explicitly as follows. The equality $\chi(\mathcal{O})=0$ holds if and only if the signature of $\mathcal{O}$ belongs to the list

$$
\{2,2,2,2\} \quad\{3,3,3\}, \quad\{2,4,4\}, \quad\{2,3,6\},
$$

while $\chi(\mathcal{O})>0$ if and only if either $\mathcal{O}$ is the non-ramified sphere or the signature of $\mathcal{O}$ belongs to the list

$$
\{n, n\}, \quad n \geq 2, \quad\{2,2, n\}, \quad n \geq 2, \quad\{2,3,3\}, \quad\{2,3,4\}, \quad\{2,3,5\} .
$$

Groups $\Gamma_{\mathcal{O}} \subset \operatorname{Aut}(\mathbb{C})$ corresponding to orbifolds $\mathcal{O}$ with signatures (14) are generated by translations of $\mathbb{C}$ by elements of some lattice $L \subset \mathbb{C}$ of rank two and the rotation $z \rightarrow \varepsilon z$, where $\varepsilon$ is an $n$th root of unity with $n$ equal to $2,3,4$, or 6 , such that $\varepsilon L=L$. Accordingly, the functions $\theta_{\mathcal{O}}$ may be written in terms of the corresponding Weierstrass functions as $\wp(z), \wp^{\prime}(z), \wp^{2}(z)$, and $\wp^{\prime 2}(z)$ (see [12], or [5. Section IV.9.5]). Groups $\Gamma_{\mathcal{O}} \subset \operatorname{Aut}\left(\mathbb{C P}^{1}\right)$ corresponding to orbifolds $\mathcal{O}$ with signatures (15) are the well-known five finite subgroups $C_{n}, D_{2 n}, A_{4}, S_{4}, A_{5}$ of $\operatorname{Aut}\left(\mathbb{C P}^{1}\right)$, and the functions $\theta_{\mathcal{O}}$ are Galois coverings of $\mathbb{C P}^{1}$ by $\mathbb{C P}^{1}$ of degrees $n$, 
$2 n, 12,24,60$, calculated for the first time by Klein in 9 . In particular, for $C_{n}$ and $D_{2 n}$ the corresponding functions $\theta_{\mathcal{O}}$ are $z^{n}$ and

$$
Z_{n}=\frac{1}{2}\left(z^{n}+\frac{1}{z^{n}}\right)
$$

2.2. Orbifolds $\mathcal{O}_{1}^{A}, \mathcal{O}_{2}^{A}$, and $\mathcal{O}_{0}^{A}$. With each rational function $A$ one can associate in a natural way two orbifolds $\mathcal{O}_{1}^{A}$ and $\mathcal{O}_{2}^{A}$ setting $\nu_{2}^{A}(z)$ equal to the least common multiple of local degrees of $f$ at the points of the preimage $A^{-1}\{z\}$, and

$$
\nu_{1}^{A}(z)=\nu_{2}^{A}(A(z)) / \operatorname{deg}_{z} A .
$$

By construction, $A: \mathcal{O}_{1}^{A} \rightarrow \mathcal{O}_{2}^{A}$ is a covering map between orbifolds. Furthermore, since the composition $A \circ \theta_{\mathcal{O}_{1}^{A}}: \widetilde{\mathcal{O}_{1}^{A}} \rightarrow \mathcal{O}_{2}^{A}$ is a covering map between orbifolds by Corollary 2.4, it follows from the uniqueness of the universal covering that

$$
\theta_{\mathcal{O}_{2}^{A}}=A \circ \theta_{\mathcal{O}_{1}^{A}} .
$$

We recall that a Lattès map can be defined as a rational function $A$ of degree at least two such that $A: \mathcal{O} \rightarrow \mathcal{O}$ is a covering map for some orbifold $\mathcal{O}$ (see [12], 20]). Such an orbifold is defined in a unique way and necessarily satisfies the condition $\chi(\mathcal{O})=0$ in view of equality (11). Following [20], we say that a rational function $A$ of degree at least two is a generalized Lattès map if there exists an orbifold $\mathcal{O}$ distinct from the non-ramified sphere such that $A: \mathcal{O} \rightarrow \mathcal{O}$ is a minimal holomorphic map between orbifolds. Notice that, similarly to usual Lattès maps, generalized Lattès maps can be described in terms of group actions and semiconjugacies (see [20]).

In general, there might be more than one orbifold $\mathcal{O}$ such that $A: \mathcal{O} \rightarrow \mathcal{O}$ is a minimal holomorphic map between orbifolds, and even infinitely many such orbifolds. Namely, the power $z^{ \pm d}: \mathcal{O} \rightarrow \mathcal{O}$ is a minimal holomorphic map for any $\mathcal{O}$ defined by the conditions

$$
\nu(0)=\nu(\infty)=n, \quad n \geq 2, \quad \operatorname{GCD}(d, n)=1,
$$

and the Chebyshev polynomial $\pm T_{d}: \mathcal{O} \rightarrow \mathcal{O}$ of degree $d$ is a minimal holomorphic map for any $\mathcal{O}$ defined by the conditions

$$
\nu(-1)=\nu(1)=2, \quad \nu(\infty)=n, \quad n \geq 1, \quad \operatorname{GCD}(d, n)=1 .
$$

Nevertheless, if $A$ is not conjugate to $z^{ \pm n}$ or $\pm T_{n}$, there exists a "maximal" orbifold $\mathcal{O}$, denoted by $\mathcal{O}_{0}^{A}$, such that $A: \mathcal{O} \rightarrow \mathcal{O}$ is a minimal holomorphic map.

In more details, for orbifolds $\mathcal{O}_{1}$ and $\mathcal{O}_{2}$ we write $\mathcal{O}_{1} \preceq \mathcal{O}_{2}$ if for any $z \in \mathbb{C} \mathbb{P}^{1}$ the condition $\nu_{1}(z) \mid \nu_{2}(z)$ holds. In this notation, the following statement holds (see [20, Theorem 1.2]).

Theorem 2.6. Let $A$ be a rational function of degree at least two not conjugate to $z^{ \pm d}$ or $\pm T_{d}$. Then there exists an orbifold $\mathcal{O}_{0}^{A}$ such that $A: \mathcal{O}_{0}^{A} \rightarrow \mathcal{O}_{0}^{A}$ is a minimal holomorphic map between orbifolds, and for any orbifold $\mathcal{O}$ such that $A: \mathcal{O} \rightarrow \mathcal{O}$ is a minimal holomorphic map between orbifolds the relation $\mathcal{O} \preceq \mathcal{O}_{0}^{A}$ holds. Furthermore, $\mathcal{O}_{0}^{A^{\circ l}}=\mathcal{O}_{0}^{A}$ for any $l \geq 1$.

Clearly, generalized Lattès maps are exactly rational functions for which the orbifold $\mathcal{O}_{0}^{A}$ is distinct from the non-ramified sphere, completed by the functions $z^{ \pm d}$ or $\pm T_{d}$ for which the orbifold $\mathcal{O}_{0}^{A}$ is not defined. Furthermore, a rational function $A$ is a Lattès map if and only if $\chi\left(\mathcal{O}_{0}^{A}\right)=0$ (see [20, Lemma 6.4]). 
2.3. Functions $A$ with $\chi\left(\mathcal{O}_{2}^{A}\right) \geq 0$. Rational functions $A$ for which the orbifold $\mathcal{O}_{2}^{A}$ has non-negative Euler characteristic play a special role in the description of solutions of (3). Below we list some properties of such functions used below.

Let $F$ and $G$ be rational functions. We will call $G$ a compositional left factor of $F$ if $F=G \circ H$ for some rational function $H$. Compositional right factors are defined in a similar way. We will say that rational functions $A_{1}$ and $A_{2}$ are $\mu$-equivalent, and write

$$
A_{1} \underset{\mu}{\sim} A_{2},
$$

if $A_{1}$ and $A_{2}$ satisfy the equality

$$
A_{1}=\mu_{1} \circ A_{2} \circ \mu_{2},
$$

for some Möbius transformations $\mu_{1}$ and $\mu_{2}$.

We recall that for a rational function $X$ its normalization $\tilde{X}$ is defined as a holomorphic function of the lowest possible degree between compact Riemann surfaces $\widetilde{X}: \widetilde{S}_{X} \rightarrow \mathbb{C P}^{1}$ such that $\widetilde{X}$ is a Galois covering and

$$
\widetilde{X}=X \circ H
$$

for some holomorphic map $H: \widetilde{S}_{X} \rightarrow \mathbb{C P}^{1}$. From the algebraic point of view the passage from $X$ to $\widetilde{X}$ corresponds to the passage from the field extension $\mathbb{C}(z) / \mathbb{C}(X)$ to its Galois closure.

In the above terms, rational functions $A$ for which $\chi\left(\mathcal{O}_{2}^{A}\right) \geq 0$ can be characterized as follows (see [18, Lemma 2.1]).

Lemma 2.7. Let $A$ be a rational function. Then $g\left(\widetilde{S}_{A}\right)=0$ if and only if $\chi\left(\mathcal{O}_{2}^{A}\right)>0$, and $g\left(\widetilde{S}_{A}\right)=1$ if and only if $\chi\left(\mathcal{O}_{2}^{A}\right)=0$.

Since, by Lemma 2.7, rational functions $A$ with $\chi\left(\mathcal{O}_{2}^{A}\right)>0$ are compositional left factors of rational Galois coverings of $\mathbb{C P}^{1}$ by $\mathbb{C P}^{1}$, these functions can be listed explicitly (see Theorem 1.1 in [18]). Below we will need only the following corollary of this classification.

Lemma 2.8. Let $A$ be a rational function of degree at least two such that $\chi\left(\mathcal{O}_{2}^{A}\right)>0$. Then either $\operatorname{deg} A$ belongs to the set

$$
E_{1}=\{4,5,6,8,10,12,15,20,24,30,60\},
$$

or $A$ is $\mu$-equivalent to one of the functions $z^{n}, T_{n}, Z_{n}$, where $n \geq 2$.

Notice that

$$
z^{2} \underset{\mu}{\sim} T_{2} \underset{\mu}{\sim} Z_{1} .
$$

However, for $n>2$ the functions $z^{n}, T_{n}$, and $Z_{n / 2}$ are pairwise not $\mu$-equivalent.

Consider now rational functions $A$ with $\chi\left(\mathcal{O}_{2}^{A}\right)=0$. Since $A: \mathcal{O}_{1}^{A} \rightarrow \mathcal{O}_{2}^{A}$ is a covering map, and $\chi\left(\mathcal{O}_{1}^{A}\right)=0$ by (11), any such a map is a covering map between orbifolds of zero Euler characteristic. In the other direction, it can be shown that if $A: \mathcal{O}_{1} \rightarrow \mathcal{O}_{2}$ is a covering map between orbifolds of zero Euler characteristic, then with a few exceptions the equalities

$$
\mathcal{O}_{1}^{A}=\mathcal{O}_{1}, \quad \mathcal{O}_{2}^{A}=\mathcal{O}_{2}
$$

hold (see [18, Theorem 5.2]). Again, we will need only the following corollary of this result. 
Lemma 2.9. Let $A$ be a rational function of degree at least two, and let $\mathcal{O}_{1}, \mathcal{O}_{2}$ be orbifolds such that $A: \mathcal{O}_{1} \rightarrow \mathcal{O}_{2}$ is a covering map between orbifolds and $\chi\left(\mathcal{O}_{1}\right)=0$, $\chi\left(\mathcal{O}_{2}\right)=0$. Then either $\operatorname{deg} A$ belongs to the set

$$
E_{2}=\{2,3,4,6,8,12\},
$$

or the equalities $\mathcal{O}_{2}=\mathcal{O}_{2}^{A}, \mathcal{O}_{1}=\mathcal{O}_{1}^{A}$ hold.

Finally, we will use the following well-known statement concerning decompositions of the functions $z^{n}, T_{n}, Z_{n}$ into a composition of two rational functions (see e.g. [18, Sections 4.1-4.2]).

\section{Lemma 2.10.}

(a) Any decomposition of $z^{n}, n \geq 2$, has the form

$$
z^{n}=\left(z^{n / d} \circ \mu\right) \circ\left(\mu^{-1} \circ z^{d}\right),
$$

where $d \mid n$ and $\mu$ is a Möbius transformation.

(b) Any decomposition of $Z_{n}, n \geq 2$, either has the form

$$
Z_{n}=\left(Z_{n / d} \circ \mu\right) \circ\left(\mu^{-1} \circ z^{d}\right),
$$

where $d \mid n$ and $\mu$ is a Möbius transformation, or has the form

$$
Z_{n}=\left(\varepsilon^{n} T_{n / d} \circ \mu\right) \circ\left(\mu^{-1} \circ Z_{d} \circ(\varepsilon z)\right),
$$

where $d \mid n, \varepsilon^{2 n}=1$, and $\mu$ is a Möbius transformation.

(c) Any decomposition of $T_{n}, n \geq 2$, has the form

$$
T_{n}=\left(T_{n / d} \circ \mu\right) \circ\left(\mu^{-1} \circ T_{d}\right),
$$

where $d \mid n$ and $\mu$ is a Möbius transformation.

2.4. Equivalence $\sim$ and special functions. Let $B$ be a rational function. For any decomposition $B=V \circ U$, where $U$ and $V$ are rational functions, the rational function $\widetilde{B}=U \circ V$ is called an elementary transformation of $B$. We say that rational functions $B$ and $A$ are equivalent and write $A \sim B$ if there exists a chain of elementary transformations between $B$ and $A$ (this equivalence relation should not be confused with the equivalence relation from Section 2.3 where the subscript $\mu$ is used). For a rational function $B$ we will denote its equivalence class by $[B]$. Since for any Möbius transformation $W$ the equality

$$
B=(B \circ W) \circ W^{-1}
$$

holds, each equivalence class $[B]$ is a union of conjugacy classes.

Equivalent functions provide examples of semiconjugate functions. Indeed, since for $B$ and $\widetilde{B}$ as above the equalities

$$
\widetilde{B} \circ U=U \circ B, \quad B \circ V=V \circ \widetilde{B}
$$

hold, $B$ is semiconjugate to $\widetilde{B}$, and $\widetilde{B}$ is semiconjugate to $B$, implying inductively that if $A \sim B$, then $A$ is semiconjugate to $B$, and $B$ is semiconjugate to $A$.

A rational function $B$ of degree at least two is called special if it is either a Lattès map, or it is conjugate to $z^{ \pm n}$ or $\pm T_{n}$. Special functions can be characterized as finite quotients of affine maps in the following sense: a rational function $B$ is special if and only if there is a lattice of rank one or two $\Lambda \subset \mathbb{C}$, an affine map $L=a t+b$ from $\mathbb{C} / \Lambda$ to $\mathbb{C} / \Lambda$, and a finite-to-one holomorphic map $\theta: \mathbb{C} / \Lambda \rightarrow \mathbb{C P}^{1} \backslash \mathcal{E}_{B}$, where 
$\mathcal{E}_{B}$ stands for the set of exceptional values of $B$, which satisfy the semiconjugacy relation

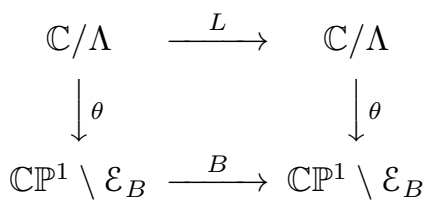

(see [12]).

Equivalently, special functions can be described as rational functions $B$ that are covering maps $B: \mathcal{O} \rightarrow \mathcal{O}$ between orbifolds for some $\mathcal{O}=(R, \nu)$ with $R=\mathbb{C P}^{1} \backslash \mathcal{E}_{B}$. It follows from (11) that for such an orbifold $\mathcal{O}$ the equality $\chi(\mathcal{O})=0$ holds, and (6) implies easily that, if $\mathcal{E}_{B} \neq \emptyset$, then either $\mathcal{E}_{B}$ contains two points and $\nu \equiv 1$, or $\mathcal{E}_{B}$ contains one point and $\nu(\mathcal{O})=\{2,2\}$. Correspondingly, the map $z^{ \pm d}: \mathcal{O} \rightarrow \mathcal{O}$ is a covering map for the non-ramified orbifold with $R=\mathbb{C P}^{1} \backslash\{0, \infty\}$, while $\pm T_{d}: \mathcal{O} \rightarrow \mathcal{O}$ is a covering map for the orbifold defined on $R=\mathbb{C P}^{1} \backslash\{\infty\}$ by the condition $\nu(1)=2, \nu(-1)=2$. The corresponding functions $\theta$ are $e^{z}$ and $\cos z$.

Below we collect several facts about special functions that we will need in the following.

Lemma 2.11. Let $F$ be a special rational function and $\widehat{F} \sim F$. Then $\widehat{F}$ is special.

Proof. Assume that $F$ is conjugate to $z^{ \pm n}$. Then, by Lemma 2.10, any elementary transformation of $F$ is conjugate to $z^{ \pm n}$, implying inductively that any $\widehat{F} \sim F$ is conjugate to $z^{ \pm n}$. If $F$ is conjugate to $\pm T_{n}$, the proof is similar. Finally, if $F$ is a Lattès, then $\widehat{F}$ is a Lattès map (see [20, Corollary 4.4]).

The next statement follows from Corollary 4.7 and Lemma 6.3 in the paper [20].

Lemma 2.12. Let $A$ be a rational function of degree $d \geq 2$ such that some iterate $A^{\circ l}, l \geq 2$, is special. Then $A$ is special.

Finally, we will need the following result (see [12, Corollary 4.3]).

Lemma 2.13. Let $A$ and $B$ be rational functions such that $A$ is semiconjugate to $B$. Then $A$ is special if and only if $B$ is special.

2.5. Good solutions of $A \circ C=D \circ B$. We say that a solution $A, C, D, B$ of the functional equation

$$
A \circ C=D \circ B
$$

in rational functions is good if the algebraic curve

$$
\mathcal{E}_{A, D}: A(x)-D(y)=0
$$

is irreducible and $\mathbb{C}(C, B)=\mathbb{C}(z)$. This definition is a particular case of the definition of good solutions of (17) in holomorphic functions defined on compact Riemann surfaces (see [14, Section 2]). In particular, Theorem 2.1 in [14] implies the following.

Lemma 2.14. If a solution $A, C, D, B$ of (17) is good, then $\operatorname{deg} C=\operatorname{deg} D$.

Furthermore, the following statement holds (see [14, Lemma 2.1]). 
Lemma 2.15. A solution $A, C, D, B$ of (17) is good whenever any two of the following three conditions are satisfied:

- the curve $\mathcal{E}_{A, D}$ is irreducible,

- the equality $\mathbb{C}(C, B)=\mathbb{C}(z)$ holds,

- the equality $\operatorname{deg} C=\operatorname{deg} D$ holds.

The property of a solution $A, C, D, B$ of (17) to be good imposes strong restrictions on the ramification collections of the functions $A, C, D, B$, which are described by the following theorem (see [14, Theorem 4.2]).

Theorem 2.16. Let $A, C, D, B$ be a good solution of (17). Then the commutative diagram

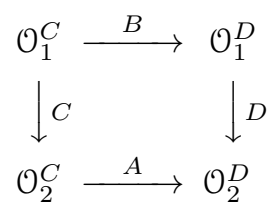

consists of minimal holomorphic maps between orbifolds.

Since an irreducible algebraic curve $\mathcal{E}_{A, D}$ has genus zero if and only if it can be parametrized by some rational functions $C$ and $B$ with $\mathbb{C}(C, B)=\mathbb{C}(z)$, describing good solutions of (17) mostly reduces to describing irreducible algebraic curves (18) of genus zero. The following general result is proved in [15].

Theorem 2.17. Let $A$ be a rational function of degree $n$ such that $\chi\left(\mathcal{O}_{2}^{A}\right)<0$. Then for any rational function $D$ of degree $m$ such that the curve $\mathcal{E}_{A, D}$ is irreducible the inequality

holds.

$$
g\left(\mathcal{E}_{A, D}\right)>\frac{m-84 n+168}{168}
$$

The practical meaning of Theorem 2.17 is that whenever $A, C, D, B$ is a good solution of (17) with

$$
\operatorname{deg} D \geq 84(\operatorname{deg} A-2),
$$

the function $A$ necessarily satisfies the restrictive condition $\chi\left(\mathcal{O}_{2}^{A}\right) \geq 0$ discussed in Section 2.3

The next result we will need states that "gluing together" two commutative diagrams corresponding to good solutions of (17) we obtain again a good solution of (17) (see the diagram below)

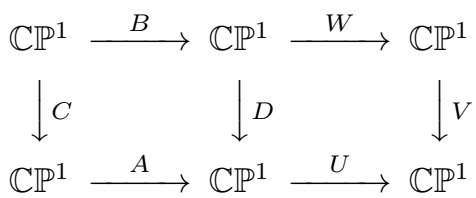

Theorem 2.18. Assume that $A, C, D, B$ and $U, D, V, W$ are good solutions of (17). Then $U \circ A, C, V, W \circ B$ is also a good solution of (17).

Proof. The theorem is a particular case of Theorem 2.10 in [21]. For the reader convenience we provide a short independent proof.

Since Lemma 2.14 implies the equalities

$$
\operatorname{deg} C=\operatorname{deg} V, \quad \operatorname{deg}(W \circ B)=\operatorname{deg}(A \circ U),
$$


it follows from Lemma 2.15 that it is enough to prove that the curve

$$
(U \circ A)(x)-V(y)=0
$$

is irreducible. Assume the inverse. Then

$$
t \rightarrow(C(t),(W \circ B)(t))
$$

is a parametrization of some proper irreducible component $F(x, y)=0$ of (20), implying that

$$
C=X \circ R, \quad W \circ B=Y \circ R,
$$

for some rational functions $X, Y$ and $R$ such that

$$
\operatorname{deg} X=\operatorname{deg}_{y} F, \quad \operatorname{deg} Y=\operatorname{deg}_{x} F .
$$

Moreover, $\operatorname{deg} R>1$ since otherwise equalities (19), (21), and (22) imply that the curve $F(x, y)=0$ coincides with (20).

Since $U, D, V, W$ is a good solution of (17), it follows from the equality

$$
(U \circ A) \circ X=U \circ(A \circ X)=V \circ Y
$$

that there exists a rational function $T$ such that

$$
A \circ X=D \circ T, \quad Y=W \circ T .
$$

Similarly, the first of these equalities implies that there exists a rational function $S$ such that

$$
X=C \circ S, \quad T=B \circ S .
$$

Thus,

$$
X=C \circ S, \quad Y=W \circ B \circ S,
$$

implying by (21) that $\operatorname{deg} R=1$. The contradiction obtained shows that (20) is irreducible.

2.6. Primitive solutions of $A \circ X=X \circ B$. We recall that a solution $A, X, B$ of equation (3) is called primitive if $\mathbb{C}(X, B)=\mathbb{C}(z)$. By Lemma 2.15, a solution $A, X, B$ of (3) is primitive if and only if the corresponding solution

$$
A=A, \quad C=X, \quad D=X, \quad B=B
$$

of (17) is good. Primitive solutions are described as follows (see [14, Theorem 6.1]).

Theorem 2.19. Let $A, B, X$ be rational functions of degree at least two such that $A \circ X=X \circ B$ and $\mathbb{C}(B, X)=\mathbb{C}(z)$. Then $\chi\left(\mathcal{O}_{1}^{X}\right) \geq 0, \chi\left(\mathcal{O}_{2}^{X}\right) \geq 0$, and the commutative diagram

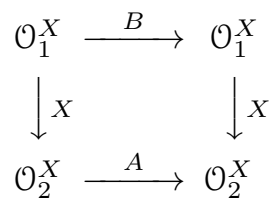

consists of minimal holomorphic maps between orbifolds.

The following statement (see [14, Theorem 5.1]) is a more precise version of Proposition 2.1 for minimal holomorphic maps $A: \mathcal{O} \rightarrow \mathcal{O}$ with $\chi(\mathcal{O})>0$. 
Theorem 2.20. Let $A$ and $F$ be rational functions of degree at least two and $\mathcal{O}$ an orbifold with $\chi(\mathcal{O})>0$ such that $A: \mathcal{O} \rightarrow \mathcal{O}$ is a holomorphic map between orbifolds and the diagram

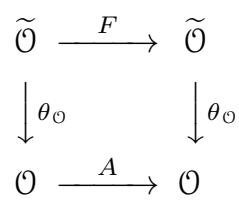

commutes. Then the following conditions are equivalent.

(1) The holomorphic map $A: \mathcal{O} \rightarrow \mathcal{O}$ is a minimal holomorphic map.

(2) The homomorphism $\varphi: \Gamma_{\mathcal{O}} \rightarrow \Gamma_{\mathcal{O}}$ defined by the equality

$$
F \circ \sigma=\varphi(\sigma) \circ F, \quad \sigma \in \Gamma_{\mathcal{O}},
$$

is an automorphism of $\Gamma_{\mathcal{O}}$.

(3) The triple $F, A, \theta_{\mathcal{O}}$ is a good solution of the equation

$$
A \circ \theta_{\mathcal{O}}=\theta_{\mathcal{O}} \circ F
$$

Primitive solution of (3) such that $X$ belongs to the series $X=z^{n}, X=T_{n}$, or $Z_{n}$ can be described explicitly (see [20, Section 5]). Below we will need only the following corollary of this description (see [20, Corollary 5.2, Corollary 5.5, and Corollary 5.8]).

Corollary 2.21. Let $A, X, B$ be a primitive solution of (3) such that $X=z^{n}$, where $n \geq 2$. Then $\operatorname{deg} A \geq n$, unless $B=c z^{ \pm m}, A=c^{n} z^{ \pm m}$. Similarly, if $X=T_{n}$, where $n>2$, then $\operatorname{deg} A \geq n+1$, unless $B= \pm T_{m}, A=( \pm 1)^{n} T_{m}$. Finally, if $X=Z_{n}$, where $n>2$, then $\operatorname{deg} A \geq n+1$, unless $B=\varepsilon z^{ \pm m}$, where $\varepsilon^{2 n}=1$, and $A=\varepsilon^{n} T_{m}$.

\section{Good Chains}

We define a chain $\mathcal{C}=\mathcal{C}(s, d)$ of length $s \geq 2$ as a sequence of $s-1$ equalities

$$
U_{i} \circ V_{i}=V_{i+1} \circ U_{i+1}, \quad 1 \leq i \leq s-1,
$$

where $U_{i}, V_{i}, 1 \leq i \leq s$, are rational functions of degree at least two. Clearly, any such a chain corresponds to a sequence of $s$ elementary transformations

$$
F \rightarrow F_{1} \rightarrow \cdots \rightarrow F_{s}
$$

where

$$
F=V_{1} \circ U_{1}, \quad F_{i}=U_{i} \circ V_{i}, \quad 1 \leq i \leq s .
$$

The function $F=V_{1} \circ U_{1}$ is called the basis of $\mathcal{C}$, and the common degree $d$ of the functions in (25) is called the degree of $\mathcal{C}$. 
Any chain (24) gives rise to the following commutative diagram:

In particular, setting

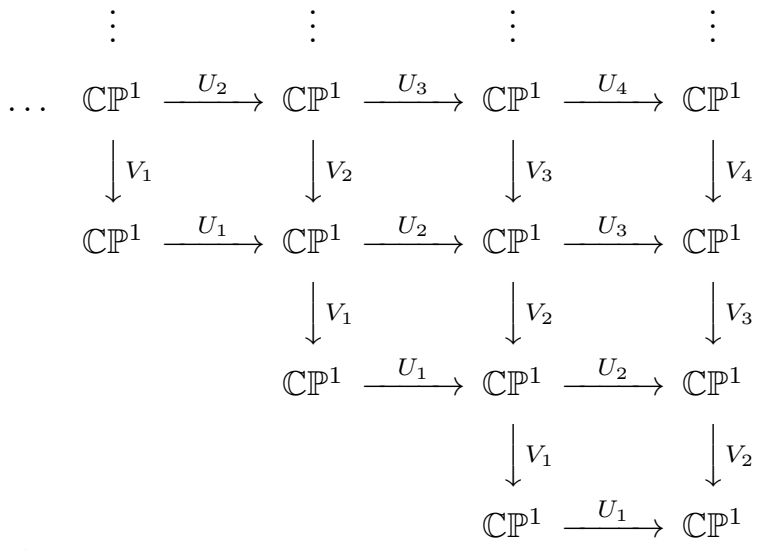

$$
V_{i, j}=V_{i} \circ V_{i+1} \circ \cdots \circ V_{j}, \quad U_{i, j}=U_{j} \circ U_{j-1} \circ \cdots \circ U_{i}, \quad 1 \leq i \leq j \leq s,
$$

we see that for any $i, j_{1}, j_{2}, 1 \leq i \leq j_{1} \leq s, 1 \leq i \leq j_{2} \leq s$, the equality

$$
U_{i, j_{1}} \circ V_{i, j_{2}}=V_{j_{1}+1, j_{1}+j_{2}-i+1} \circ U_{j_{2}+1, j_{1}+j_{2}-i+1}
$$

holds. Furthermore, the following statement is true (see [20, Lemma 3.1]).

Lemma 3.1. Let $\mathcal{C}$ be a chain given by (24) and (25). Then

$$
V_{1, s} \circ U_{1, s}=F^{\circ s}, \quad U_{1, s} \circ V_{1, s}=F_{s}^{\circ s} \text {. }
$$

Let $\mathcal{C}=\mathcal{C}(s, d)$ be a chain. We define its dual chain $\widehat{\mathcal{C}}$ by the formulas

$$
\widehat{U}_{i}=V_{s+1-i}, \quad \widehat{V}_{i}=U_{s+1-i}, \quad 1 \leq i \leq s .
$$

For a natural number $k$ such that

$$
l_{k}=[s / k] \geq 2
$$

we define $\mathcal{C}_{k}=\mathcal{C}\left(l_{k}, d^{k}\right)$ as a chain corresponding to the sequence of $l_{k}-1$ equalities

$$
\begin{gathered}
U_{1, k} \circ V_{1, k}=V_{k+1,2 k} \circ U_{k+1,2 k}, \\
U_{k+1,2 k} \circ V_{k+1,2 k}=V_{2 k+1,3 k} \circ U_{2 k+1,3 k}, \\
U_{2 k+1,3 k} \circ V_{2 k+1,3 k}=V_{3 k+1,4 k} \circ U_{3 k+1,4 k}, \\
\ldots \\
U_{\left(l_{k}-2\right) k+1,\left(l_{k}-1\right) k} \circ V_{\left(l_{k}-2\right) k+1,\left(l_{k}-1\right) k}=V_{\left(l_{k}-1\right) k+1, l_{k} k} \circ U_{\left(l_{k}-1\right) k+1, l_{k} k} .
\end{gathered}
$$

A chain $\mathcal{C}=\mathcal{C}(s, d)$ is called good if all solutions of (17) provided by equalities (24) are good. For a good chain $\mathcal{C}$ set

$$
d_{1}=\operatorname{deg} U_{1}=\operatorname{deg} U_{2}=\cdots=\operatorname{deg} U_{s},
$$

and

$$
d_{2}=\operatorname{deg} V_{1}=\operatorname{deg} V_{2}=\cdots=\operatorname{deg} V_{s} .
$$

These numbers are well defined by Lemma 2.14, and obviously satisfy the equality $d_{1} d_{2}=d$. For good chains we will use the notation $\mathcal{C}=\mathcal{C}\left(s, d_{1}, d_{2}\right)$ instead of the notation $\mathcal{C}=\mathfrak{C}(s, d)$. Clearly, Theorem 2.18 implies inductively the following statement. 
Lemma 3.2. Let $\mathcal{C}=\mathcal{C}\left(s, d_{1}, d_{2}\right)$ be a good chain. Then any solution of (17) of the form (26) is good.

In this section we prove one of the main results of the paper: the finiteness of any good chain whose basis is non-special. More precisely, we prove the following.

Theorem 3.3. Let $\mathcal{C}$ be a good chain of length $s$ and degree $d$ with non-special basis. Then $s<12 \log _{2} d+11$.

Notice that good chains of length $\approx \log _{2} d$ with non-special bases $F$ of degree $d$ exist and are easy to construct (see [14, p. 1241]). On the other hand, for special $F$ the theorem is not true. Indeed, taking any commuting pair $A, B$ of powers, Chebyshev polynomials, or Lattès maps such that $\mathbb{C}(A, B)=\mathbb{C}(z)$, and setting $U_{i}=A, V_{i}=B, i \geq 1$, we obviously obtain an infinite good chain.

Before proving Theorem 3.3 we will prove the following two lemmas.

Lemma 3.4. Let $A, C, D, B$ be a good solution of (17) such that either $C \underset{\mu}{\sim} T_{n}$ and $D \underset{\mu}{\sim} Z_{n / 2}$, or $C \underset{\mu}{\sim} Z_{n / 2}$ and $D \underset{\mu}{\sim} z^{n}$. Then $n \leq 2$.

Proof. Assume that $C \underset{\mu}{\sim} T_{n}$ and $D \underset{\mu}{\sim} Z_{n / 2}$. By Theorem 2.16, $B: \mathcal{O}_{1}^{C} \rightarrow \mathcal{O}_{1}^{D}$ is a minimal holomorphic map between orbifolds. On the other hand, if $n>2$, then $\nu\left(\mathcal{O}_{1}^{C}\right)=\{2,2\}$, while the orbifold $\nu\left(\mathcal{O}_{1}^{D}\right)$ is non-ramified. Since for such $\nu\left(\mathcal{O}_{1}^{C}\right)$ and $\nu\left(\mathcal{O}_{1}^{D}\right)$ condition obviously (13) is not satisfied at points $z$ where $\nu_{\mathcal{O}_{1}^{C}}(z)=2$, we conclude that $n \leq 2$ (in which case $\mathcal{O}_{1}^{C}$ is non-ramified).

Assume now that $C \underset{\mu}{\sim} Z_{n / 2}$ and $D \underset{\mu}{\sim} z^{n}$. In this case $C$ and $D$ are the universal coverings of the orbifolds $\mathcal{O}_{2}^{C}$ and $\mathcal{O}_{2}^{D}$, so that $\Gamma_{\mathcal{O}_{2}^{C}}=D_{n}$ and $\Gamma_{\mathcal{O}_{2}^{D}}=C_{n}$. Since $A: \mathcal{O}_{2}^{C} \rightarrow \mathcal{O}_{2}^{D}$ is a minimal holomorphic map between orbifolds by Theorem 2.16, it follows from Proposition 2.1 that there exists a homomorphism $\varphi: \Gamma_{\mathcal{O}_{2}^{C}} \rightarrow \Gamma_{\mathcal{O}_{2}^{D}}$ such that

$$
B \circ \sigma=\varphi(\sigma) \circ B, \quad \sigma \in \Gamma_{\mathcal{O}_{2}^{C}} .
$$

Moreover, if $n>2$, then $\operatorname{Ker}(\varphi) \neq e$, since $\left|D_{n}\right|=\left|C_{n}\right|$ but the groups $D_{n}$ and $C_{n}$ are not isomorphic. On the other hand, $\operatorname{Ker}(\varphi)$, as any other subgroup of $\Gamma_{\mathcal{O}_{2}^{C}}$, has the form $\Gamma_{\mathcal{O}^{\prime}}$ for some orbifold $\mathcal{O}^{\prime}$. Clearly, the both functions $C$ and $B$ are invariant with respect to $\Gamma_{\mathcal{O}^{\prime}}$, implying that they are rational functions in $\theta_{\mathcal{O}^{\prime}}$. Therefore, since $\Gamma_{\mathcal{O}^{\prime}} \neq e$ implies that $\operatorname{deg} \theta_{\mathcal{O}^{\prime}}>1$, the quadruple $A, C, D, B$ is not a good solution of (17). This contradiction shows that $n \leq 2$.

We recall that the sets $E_{1}$ and $E_{2}$ are defined in Lemma 2.8 and Lemma 2.9

Lemma 3.5. Let $A$ be a rational function such that $\chi\left(\mathcal{O}_{2}^{A}\right)=0$, and $U, V$ rational functions of degree at least two such that $A=U \circ V$ and $\operatorname{deg} U, \operatorname{deg} V \notin E_{2}$. Then

$$
\mathcal{O}_{1}^{A}=\mathcal{O}_{1}^{V}, \quad \mathcal{O}_{2}^{V}=\mathcal{O}_{1}^{U}, \quad \mathcal{O}_{2}^{A}=\mathcal{O}_{2}^{U}, \quad \chi\left(\mathcal{O}_{2}^{V}\right)=0 .
$$

Proof. Since $A: \mathcal{O}_{1}^{A} \rightarrow \mathcal{O}_{2}^{A}$ is a covering map between orbifolds, the maps

$$
V: \mathcal{O}_{1}^{A} \rightarrow U^{*} \mathcal{O}_{2}^{A}, \quad U: U^{*} \mathcal{O}_{2}^{A} \rightarrow \mathcal{O}_{2}^{A}
$$

are covering maps by Corollary 2.5. In particular, applying (11) to the second map in (28), we conclude that

$$
\chi\left(U^{*} \mathcal{O}_{2}^{A}\right)=0 .
$$


It follows now from Lemma 2.9 applied to covering maps (28) that

$$
\mathcal{O}_{1}^{A}=\mathcal{O}_{1}^{V}, \quad U^{*} \mathcal{O}_{2}^{A}=\mathcal{O}_{2}^{V},
$$

and

implying that

$$
U^{*} \mathcal{O}_{2}^{A}=\mathcal{O}_{1}^{U}, \quad \mathcal{O}_{2}^{A}=\mathcal{O}_{2}^{U}
$$

$$
\mathcal{O}_{2}^{V}=\mathcal{O}_{1}^{U}
$$

Finally, since $A: \mathcal{O}_{1}^{A} \rightarrow \mathcal{O}_{2}^{A}$ is a covering map, $\chi\left(\mathcal{O}_{1}^{A}\right)=0$ by (11). Thus, the first equality in (27) implies that $\chi\left(\mathcal{O}_{1}^{V}\right)=0$, and applying (11) again we see that $\chi\left(\mathcal{O}_{2}^{V}\right)=0$.

Proof of Theorem 3.3. Assume first that $d_{1} \geq d_{2}$ and, in addition, that $d_{1}$ is not contained in the set $E_{2}$ from Lemma 2.9. We show that in this case

$$
s<\log _{d_{2}} 84\left(d_{1}^{3}-2\right)+3,
$$

unless the basis $F$ of $\mathcal{C}$ is a special function.

Let us consider the equality

$$
U_{1,3} \circ V_{1, s-3}=V_{4, s} \circ U_{s-2, s} .
$$

Since

if

$$
\operatorname{deg} U_{1,3}=d_{1}^{3}, \quad \operatorname{deg} V_{1, s-3}=d_{2}^{s-3},
$$

then

$$
s \geq \log _{d_{2}} 84\left(d_{1}^{3}-2\right)+3,
$$

$$
\operatorname{deg} V_{1, s-3}=d_{2}^{s-3} \geq 84\left(d_{1}^{3}-2\right)=84\left(\operatorname{deg} U_{1,3}-2\right) .
$$

Thus, since the solution of (17) provided by equality (30) is good by Lemma 3.2. it follows from Theorem 2.17 that $\chi\left(\mathcal{O}_{2}^{U_{1,3}}\right) \geq 0$.

Assume first that $\chi\left(\mathcal{O}_{2}^{U_{1,3}}\right)>0$. Since $d_{1} \notin E_{2}$ implies that $d_{1} \neq 2$, and $d_{1} \neq 2$ implies that $d_{1}^{3}$ is not contained in the set $E_{1}$, Lemma 2.8 implies that $U_{1,3}$ is $\mu$ equivalent either to $z^{d_{1}^{3}}$, or $T_{d_{1}^{3}}$, or $Z_{d_{1}^{3} / 2}$. In case if $U_{1,3}$ is $\mu$-equivalent to $z^{d_{1}^{3}}$, Lemma 2.10 applied to the decompositions

$$
U_{1,3}=U_{2,3} \circ U_{1}, \quad U_{1,3}=U_{3} \circ U_{1,2}
$$

implies that

$$
\begin{aligned}
& U_{2,3}=\mu_{2} \circ z^{d_{1}^{2}} \circ \nu_{1}^{-1}, \quad U_{1}=\nu_{1} \circ z^{d_{1}} \circ \mu_{1}, \\
& U_{3}=\mu_{2} \circ z^{d_{1}} \circ \nu_{2}^{-1}, \quad U_{1,2}=\nu_{2} \circ z^{d_{1}^{2}} \circ \mu_{1},
\end{aligned}
$$

for some Möbius transformations $\mu_{1}, \mu_{2}, \nu_{1}, \nu_{2}$. Clearly, $d_{1} \geq d_{2}$ implies that $d_{1}^{2}>d_{2}$. Furthermore, the solution of (17) provided by the equality

$$
U_{1,2} \circ V_{1}=V_{3} \circ U_{2,3}
$$

is good by Lemma 3.2. Therefore, since

$$
U_{1,2} \underset{\mu}{\sim} z^{d_{1}^{2}}, \quad U_{2,3} \underset{\mu}{\sim} z^{d_{1}^{2}},
$$

we can apply Corollary 2.21 to (31), concluding that

$$
V_{1}=\mu_{1}^{-1} \circ c z^{ \pm d_{2}} \circ \nu_{1}^{-1}, \quad V_{3}=\nu_{2} \circ c^{d_{1}^{2}} z^{ \pm d_{2}} \circ \mu_{2}^{-1},
$$

for some $c \in \mathbb{C}$. Thus,

$$
F_{1}=U_{1} \circ V_{1}=\nu_{1} \circ c^{d_{1}} z^{ \pm d_{1} d_{2}} \circ \nu_{1}^{-1}
$$


is conjugate to $z^{ \pm d_{1} d_{2}}$. Since $F_{1} \sim F$, this implies by Lemma 2.11 that $F$ is special. Similarly, if $U_{1,3}$ is $\mu$-equivalent to $T_{d_{1}^{3}}$ we conclude that $F$ is conjugate to $\pm T_{d_{1} d_{2}}$.

Finally, the assumption that $U_{1,3}$ is $\mu$-equivalent to $Z_{d_{1}^{3} / 2}$ leads to a contradiction. Indeed, in this case applying Lemma 2.10 inductively to the decomposition

$$
U_{1,3}=U_{3} \circ U_{2} \circ U_{1}
$$

we conclude that either

$$
U_{3} \underset{\mu}{\sim} Z_{d_{1} / 2}, \quad U_{2} \underset{\mu}{\sim} z^{d_{1}}, \quad U_{1} \underset{\mu}{\sim} z^{d_{1}},
$$

or

or

$$
U_{3} \underset{\mu}{\sim} T_{d_{1}}, \quad U_{2} \underset{\mu}{\sim} T_{d_{1}}, \quad U_{1} \underset{\mu}{\sim} Z_{d_{1} / 2},
$$

$$
U_{3} \underset{\mu}{\sim} T_{d_{1}}, \quad U_{2} \underset{\mu}{\sim} Z_{d_{1} / 2}, \quad U_{1} \underset{\mu}{\sim} z^{d_{1}} .
$$

Since the solution of (17) provided by the equality

$$
V_{3} \circ U_{3}=U_{2} \circ V_{2}
$$

is good, in the first case applying the second part of Lemma 3.4 to (32) we obtain a contradiction with $d_{1} \neq 2$. Similarly, in the second case, we obtain a contradiction applying the first part of Lemma 3.4 to the equality

$$
V_{2} \circ U_{2}=U_{1} \circ V_{1} \text {. }
$$

Lastly, in the third case we obtain a contradiction applying Lemma 3.4 to either of equalities (32), (33).

Assume now that $\chi\left(\mathcal{O}_{2}^{U_{1,3}}\right)=0$. Since $d_{1} \notin E_{2}$ implies $d_{1}^{2} \notin E_{2}$, it follows from Lemma 3.5 applied to the decomposition $U_{1,3}=U_{3} \circ U_{1,2}$ that

$$
\chi\left(\mathcal{O}_{2}^{U_{1,2}}\right)=0 .
$$

Applying now Lemma 3.5 to the decomposition $U_{1,2}=U_{2} \circ U_{1}$, we obtain the equalities

$$
\begin{gathered}
\mathcal{O}_{2}^{U_{1}}=\mathcal{O}_{1}^{U_{2}}, \\
\mathcal{O}_{2}^{U_{1,2}}=\mathcal{O}_{2}^{U_{2}}, \\
\chi\left(\mathcal{O}_{2}^{U_{1}}\right)=0 .
\end{gathered}
$$

Clearly, (34) and (36) imply that

$$
\chi\left(\mathcal{O}_{2}^{U_{2}}\right)=0 .
$$

Further, since Theorem 2.16 applied to (33) implies that $V_{2}: \mathcal{O}_{2}^{U_{2}} \rightarrow \mathcal{O}_{2}^{U_{1}}$ is a minimal holomorphic map between orbifolds, it follows from equalities (37) and (38) by Proposition 2.2 that $V_{2}: \mathcal{O}_{2}^{U_{2}} \rightarrow \mathcal{O}_{2}^{U_{1}}$ is a covering map between orbifolds. Since $U_{2}: \mathcal{O}_{1}^{U_{2}} \rightarrow \mathcal{O}_{2}^{U_{2}}$ also is a covering map, it follows now from Corollary 2.4 that

$$
F_{1}=V_{2} \circ U_{2}: \mathcal{O}_{1}^{U_{2}} \rightarrow \mathcal{O}_{2}^{U_{1}}
$$

is a covering map too. Therefore, in view of equality (35), the function $F_{1}$ is a Lattès map, implying by Lemma 2.11 that the function $F$ is special.

We proved that under the assumptions $d_{1} \geq d_{2}$ and $d_{1} \notin E_{2}$ equality (29) holds, unless $F$ is a special function. Let us explain now how to get rid of these assumptions and obtain the inequality from the formulation of the theorem. First, 
if $d_{1}$ is contained in the set $E_{2}$, we can consider instead of the chain $\mathcal{C}=\mathcal{C}\left(s, d_{1}, d_{2}\right)$ the chain $\mathcal{C}_{4}=\mathcal{C}\left(\left[\frac{s}{4}\right], d_{1}^{4}, d_{2}^{4}\right)$, which is also good by Lemma 3.2 Since for any number $d_{1} \geq 2$ the number $d_{1}^{4}$ does not belong to $E_{2}$, the above argument shows that

$$
\left[\frac{s}{4}\right]<\log _{d_{2}^{4}} 84\left(d_{1}^{12}-2\right)+3,
$$

unless the basis $\widetilde{F}$ of $\mathcal{C}_{4}$ is a special function. On the other hand, since

$$
\widetilde{F}=V_{1,4} \circ U_{1,4}=F^{\circ 4}
$$

by Lemma 3.1, it follows from Lemma 2.12 that if $\widetilde{F}$ is a special function, then $F$ also is a special function.

Furthermore, since

$$
d_{2}^{4} \geq 2^{4}, \quad d_{1}^{12} \leq\left(\frac{d}{2}\right)^{12}
$$

and (39) implies that

$$
\frac{s}{4}<\log _{d_{2}^{4}} 84\left(d_{1}^{12}-2\right)+4,
$$

we conclude that inequality (39) yields the inequality

$$
\begin{gathered}
s<4 \log _{d_{2}^{4}} 84\left(d_{1}^{12}-2\right)+16 \leq \log _{2} 84\left(\left(\frac{d}{2}\right)^{12}-2\right)+16 \\
<\log _{2} 84+\log _{2}\left(\frac{d}{2}\right)^{12}+16<12 \log _{2} d+11
\end{gathered}
$$

from the formulation of the theorem. Thus, if the inequality

$$
s<12 \log _{2} d+11
$$

does not hold, inequality (39) does not hold either, implying that $F$ is special.

Finally, if $d_{1}<d_{2}$, we can consider instead of the chain $\mathcal{C}=\mathcal{C}\left(s, d_{1}, d_{2}\right)$ its dual chain $\widehat{\mathrm{C}}=\widehat{\mathrm{C}}\left(s, d_{2}, d_{1}\right)$. By the above argument, inequality (40) holds, unless the basis $\widehat{F}$ of $\widehat{\mathcal{C}}$ is a special function. On the other hand, since

$$
\widehat{F}=U_{s} \circ V_{s} \sim F,
$$

if the basis $\widehat{F}$ of $\widehat{\mathcal{C}}$ is a special function, then the basis $F$ of $\mathcal{C}$ is also a special function by Lemma 2.11

\section{Group $G(B)$ And primitive solutions}

For a rational function $B$, we denote by $\mathcal{E}(B)$ the set of rational functions $X$ of degree at least two such that (3) holds for some rational function $A$, and by $\mathcal{E}_{0}(B)$ the subset of $\mathcal{E}(B)$ consisting of functions $X$ such that $\mathbb{C}(X, B)=\mathbb{C}(z)$. In addition, we denote by $\overline{\mathcal{E}_{0}(B)}$ the quotient set of $\mathcal{E}_{0}(B)$ by the equivalence relation which identifies $X_{1}, X_{2} \in \mathcal{E}_{0}(B)$ if there exists a Möbius transformation $\mu$ such that

$$
X_{1}=\mu \circ X_{2} .
$$

In this section, we prove the finiteness of the set $\overline{\mathcal{E}_{0}(B)}$ for non-special $B$. Abusing the notation we will denote by $X$ both an element of $\mathcal{E}_{0}(B)$ and its equivalence class in $\overline{\varepsilon_{0}(B)}$. 
We recall that the symmetry group of a rational function $F$ is defined as the group of all Möbius transformations $\mu$ commuting with $F$. Since such transformations map periodic points of $F$ of any given period to themselves and any Möbius transformation is defined by its values at any three points, the symmetry group of any rational function is finite. We define the extended symmetry group $G(F)$ of $F$ as the group of Möbius transformations $\sigma$ such that

$$
F \circ \sigma=\nu_{\sigma} \circ F
$$

for some Möbius transformations $\nu_{\sigma}$. It is easy to see that $G(F)$ is indeed a group with respect to the composition operation and that the map

$$
\gamma_{F}: \sigma \rightarrow \nu_{\sigma}
$$

is a homomorphism from $G(F)$ to the group $\operatorname{Aut}\left(\mathbb{C P}^{1}\right)$.

We denote by $\mathcal{D}$ the subgroup of $\operatorname{Aut}\left(\mathbb{C P}^{1}\right)$ consisting of the transformations $\sigma=c z^{ \pm 1}, c \in \mathbb{C} \backslash\{0\}$. Notice that $\mathcal{D}$ can be described as the subgroup of Aut $\left(\mathbb{C P}^{1}\right)$ consisting of all Möbius transformations $\sigma$ such that $\sigma\{0, \infty\}=\{0, \infty\}$, or equivalently such that $\sigma^{-1}\{0, \infty\}=\{0, \infty\}$.

Lemma 4.1. For $F=z^{ \pm d}$ the group $G(F)$ is $\mathcal{D}$.

Proof. It is clear that $\mathcal{D} \subseteq G(F)$. On the other hand, if equality (41) holds, then $\nu_{\sigma}^{-1}\{0, \infty\}=\{0, \infty\}$, since otherwise the preimage $\left(\nu_{\sigma} \circ F\right)^{-1}\{0, \infty\}$ and hence the preimage $(F \circ \sigma)^{-1}\{0, \infty\}$ contains more than two points. Therefore, for any $\sigma \in G(F)$ the transformation $\nu_{\sigma}$ belongs to $\mathcal{D}$. Now (41) implies that $\sigma$ also belongs to $\mathcal{D}$.

Theorem 4.2. Let $F$ be a rational function of degree $d \geq 2$ such that $F \ngtr z^{d}$. Then the group $G(F)$ is isomorphic to one of the five finite rotation groups of the sphere $A_{4}, S_{4}, A_{5}, C_{n}, D_{2 n}$, and the order of any element of $G(F)$ does not exceed d. In particular, $|G(F)| \leq \max \{60,2 d\}$.

Proof. Any non-identical element of the group $\operatorname{Aut}\left(\mathbb{C P}^{1}\right) \cong \mathrm{PSL}_{2}(\mathbb{C})$ is conjugate either to $z \rightarrow z+1$ or to $z \rightarrow \lambda z$ for some $\lambda \in \mathbb{C} \backslash\{0,1\}$. Thus, making the change

$$
F \rightarrow \mu_{1} \circ F \circ \mu_{2}, \quad \sigma \rightarrow \mu_{2}^{-1} \circ \sigma \circ \mu_{2}, \quad \nu_{\sigma} \rightarrow \mu_{1} \circ \nu_{\sigma} \circ \mu_{1}^{-1}
$$

for convenient $\mu_{1}, \mu_{2} \in \operatorname{Aut}\left(\mathbb{C P}^{1}\right)$, without loss of generality we may assume that $\sigma$ and $\nu_{\sigma}$ in (41) have one of the two forms above.

We observe first that the equalities

$$
F(z+1)=\lambda F(z), \quad \lambda \in \mathbb{C} \backslash\{0,1\},
$$

and

$$
F(z+1)=F(z)+1
$$

are impossible. Indeed, if $F$ has a finite pole, then any of these equalities implies that $F$ has infinitely many poles. On the other hand, if $F$ is a polynomial of degree $d \geq 2$, then we obtain a contradiction comparing the coefficients of $z^{d}$ in the left and the right sides of (42), and the coefficients of $z^{d-1}$ in left and the right sides of (43), correspondingly.

Furthermore, comparing the free terms in the Laurent series at infinity of the left and the right sides of the equality

$$
F(\lambda z)=F(z)+1, \quad \lambda \in \mathbb{C} \backslash\{0,1\},
$$


we conclude that this equality is impossible either. Thus,

$$
F\left(\lambda_{1} z\right)=\lambda_{2} F(z), \quad \lambda_{1}, \lambda_{2} \in \mathbb{C} \backslash\{0,1\} .
$$

Comparing now coefficients in the left and the right sides of (44) and taking into account that $F \underset{\mu}{\chi_{\mu}} z^{d}$, we conclude that $\lambda_{1}$ is a root of unity. Furthermore, the order of the transformation $z \rightarrow \lambda_{1} z$ in the group $G(F)$ does not exceed the maximum number $n$ such that $F$ can be represented in the form

$$
F=z^{r} R\left(z^{n}\right), \quad R \in \mathbb{C}(z) .
$$

In particular, the order of any element of $G(F)$ does not exceed $d$. Indeed, since $F \nsim z^{d}$, the function $R$ in (45) has a zero or a pole distinct from 0 and $\infty$, implying $\mu$ that $d \geq n$.

The above argument shows that any element of $G(F)$ has finite order. In order to prove the finiteness of $G(F)$ we will use the Schur theorem, which states that if any element of a finitely generated subgroup $G$ of $\mathrm{GL}_{k}(\mathbb{C})$ has finite order, then $G$ has finite order (see e.g. [2, (36.2)]). Specifically, assume that $G(F)$ is infinite, and let $\sigma_{1}, \sigma_{2}, \ldots, \sigma_{s}, \ldots$ be an infinite sequence of pairwise distinct elements of $G(F)$. Observe first that for any $s \geq 1$ the finitely generated group

$$
\Gamma_{s}=<\sigma_{1}, \sigma_{2}, \ldots, \sigma_{s}>
$$

is finite. Indeed, if $\Gamma_{s}$ is infinite, then its lifting

$$
\widehat{\Gamma}_{s} \subset \mathrm{SL}_{2}(\mathbb{C}) \subset \mathrm{GL}_{2}(\mathbb{C})
$$

is also infinite, implying by the Schur theorem that $\widehat{\Gamma}_{s}$ has an element of infinite order. But in this case $\Gamma_{s}$ also has an element of infinite order in contradiction with the fact that any element of $G(F)$ has finite order.

Since the elements $\sigma_{1}, \sigma_{2}, \ldots, \sigma_{s}, \ldots$ are pairwise distinct, $\left|\Gamma_{s}\right| \rightarrow \infty$. On the other hand, since the groups $\Gamma_{s}, s \geq 1$, are finite subgroups of $\operatorname{Aut}\left(\mathbb{C P}^{1}\right)$, they belong to the list $A_{4}, S_{4}, A_{5}, C_{n}, D_{2 n}$. Therefore, for $s$ big enough the group $\Gamma_{s}$ is either $C_{n}$ or $D_{2 n}$, where $n$ satisfies the inequality $n>d$. However, since the both groups $C_{n}$ and $D_{2 n}$ have an element of order $n$, this contradicts to the fact that the order of any element of $G(F)$ does not exceed $d$. Thus, the group $G(F)$ is finite. Finally, if $G(F)$ is $A_{4}, S_{4}$, or $A_{5}$, then $G(F) \leq 60$, while if $G(F)$ is $C_{n}$ or $D_{2 n}$, then $n \leq d$, since $C_{n}$ and $D_{2 n}$ have an element of order $n$.

Lemma 4.3. Let $\delta$ be a Möbius transformation that does not belong to $\mathcal{D}$. Then the intersection of the groups $\mathcal{D}$ and $\delta^{-1} \circ \mathcal{D} \circ \delta$ is a finite group isomorphic to a subgroup of the Klein four-group.

Proof. Let us denote the intersection of the groups $\mathcal{D}$ and $\delta^{-1} \circ \mathcal{D} \circ \delta$ by $\mathcal{R}$. We show first that $\mathcal{R}$ consists of involutions. Assume in contrary that $\mu \in \mathcal{R}$ is not an involution, and let $\mu^{\prime}$ be an element of $\mathcal{D}$ which makes the diagram

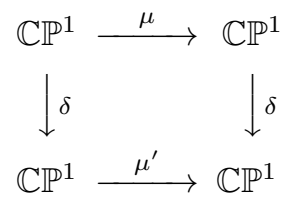


commutative. Since the diagram

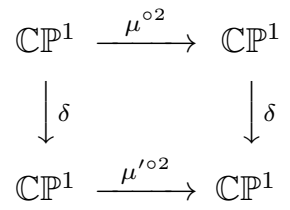

also is commutative, $\mu^{\prime}$ is not an involution either. Therefore, since

$$
\mu\{0, \infty\}=\{0, \infty\}, \quad \mu^{\prime}\{0, \infty\}=\{0, \infty\},
$$

the set $\{0, \infty\}$ is the fixed point set of the transformations $\mu^{\circ 2}$ and $\mu^{\prime \circ 2}$. It follows now from (46) that $\delta\{0, \infty\}=\{0, \infty\}$, in contradiction with $\delta \notin \mathcal{D}$.

Since $\mathcal{R}$ consists of involutions, any $\mu \in \mathcal{R}$ has either the form $\mu= \pm z$, or the form $\mu=c / z, c \in \mathbb{C} \backslash\{0\}$. Furthermore, if two transformations $\mu_{1}=c_{1} / z$, $c_{1} \in \mathbb{C} \backslash\{0\}$, and $\mu_{2}=c_{2} / z, c_{2} \in \mathbb{C} \backslash\{0\}$, belong to $\mathcal{R}$, then their composition $\frac{c_{1}}{c_{2}} z$ also belongs to $\mathcal{R}$ implying that $c_{2}= \pm c_{1}$. Therefore, the group $\mathcal{R}$ contains at most four elements: $\pm z, \pm \frac{c_{1}}{z}$.

Theorem 4.4. Let $B$ be a non-special rational function of degree $d \geq 2$. Then for any $X \in \mathcal{E}_{0}(B)$ the inequalities $\chi\left(\mathcal{O}_{2}^{X}\right)>0$ and $\operatorname{deg} X \leq \max \{60,2 d\}$ hold. Furthermore, the set $\overline{\mathcal{E}_{0}(B)}$ is finite and its cardinality can be bounded from above by a number depending on d only.

Proof. Let $X$ be an element of $\mathcal{E}_{0}(B)$ and $A$ the corresponding function such that (3) holds. By Theorem 2.19, the diagram

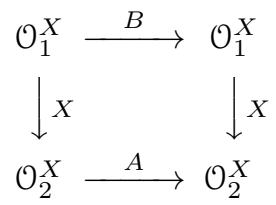

consists of minimal holomorphic maps between orbifolds, and $\chi\left(\mathcal{O}_{2}^{X}\right) \geq 0$. Moreover, in fact $\chi\left(\mathcal{O}_{2}^{X}\right)>0$. Indeed, if $\chi\left(\mathcal{O}_{2}^{X}\right)=0$, then $\chi\left(\mathcal{O}_{1}^{X}\right)=0$ by (11). Therefore, since

$$
B: \mathcal{O}_{1}^{X} \rightarrow \mathcal{O}_{1}^{X}
$$

is a minimal holomorphic map, it follows from Proposition 2.2 that (48) is a covering map, implying that $B$ is a Lattès map, in contradiction with the assumption. Notice that $\chi\left(\mathcal{O}_{2}^{X}\right)>0$ implies $\chi\left(\mathcal{O}_{1}^{X}\right)>0$ by (11).

Since $B$ is non-special and (48) is a minimal holomorphic map, it follows from Theorem 2.6] that the orbifold $\mathcal{O}_{0}^{B}$ is defined and $\mathcal{O}_{1}^{X} \preceq \mathcal{O}_{0}^{B}$. Moreover, since $B$ is not a Lattès map, $\chi\left(\mathcal{O}_{0}^{B}\right)>0$. We observe first that the number of orbifolds $\mathcal{O}$ such that $\mathcal{O} \preceq \mathcal{O}_{0}^{B}$ is finite and can be bounded by a number depending on $d$ only. Clearly, it is enough to show that if $\mathcal{O}_{0}^{B}$ belongs to the series $\{n, n\}, n \geq 2$, or $\{2,2, n\}, n>2$, then $n$ is bounded in terms of $d$. By Theorem 2.20, there exists a rational function $F_{0}$ such that the diagram

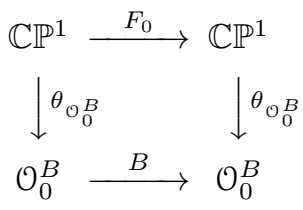


commutes and the triple $B, \theta_{\mathcal{O}_{0}^{B}}, F_{0}$ is a primitive solution of equation (3). By condition, $B$ is not special. Therefore, if $\theta_{\mathcal{O}_{0}^{B}}$ is $\mu$-equivalent to $z^{n}$ or to $Z_{n}$, applying Corollary 2.21 to (49) we conclude that $n \leq d$.

Since the number of orbifolds $\mathcal{O}$ such that $\mathcal{O} \preceq \mathcal{O}_{0}^{B}$ is finite, in order to prove the finiteness of $\overline{\varepsilon_{0}(B)}$ it is enough to show that for any fixed orbifold $\mathcal{O}$ with $\chi(\mathcal{O})>0$ there exist only finitely many $X \in \overline{\mathcal{E}_{0}(B)}$ such that $\mathcal{O}_{1}^{X}=\mathcal{O}$. Assume first that $\mathcal{O}$ is non-ramified. Then $X=\theta_{\mathcal{O}_{2}^{X}}$ by (16), and diagram (47) reduces to diagram (23). Furthermore, by Theorem 2.20 there exists an automorphism $\varphi: \Gamma_{\mathcal{O}_{2}^{X}} \rightarrow \Gamma_{\mathcal{O}_{2}^{X}}$ such that for any $\sigma \in \Gamma_{\mathcal{O}_{2}^{X}}$ the equality

$$
B \circ \sigma=\varphi(\sigma) \circ B
$$

holds. Therefore, $\Gamma_{\mathcal{O}_{2}^{X}}$ belongs to the intersection

$$
G_{0}(B)=G(B) \cap \gamma_{B}(G(B)),
$$

and hence the number of classes $X$ in $\overline{\mathcal{E}_{0}(B)}$ with non-ramified $\mathcal{O}_{1}^{X}$ does not exceed the number of subgroups of $G_{0}(B)$. Since $G_{0}(B) \subseteq G(B)$ and

$$
|G(B)| \leq \max \{60,2 d\}
$$

whenever $B \underset{\mu}{\nsim} z^{d}$, we conclude that for $B \underset{\mu}{\chi} z^{d}$ the number of subgroups of $G_{0}(B)$ and therefore the cardinality of $\overline{\mathcal{E}_{0}(B)}$ is bounded from above by a number depending on $d$ only. Moreover,

$$
\operatorname{deg} X=\operatorname{deg} \theta_{\mathcal{O}_{2}^{X}}=\left|\Gamma_{\mathcal{O}_{2}^{X}}\right| \leq|G(B)| \leq \max \{60,2 d\} .
$$

Assume now that $B \underset{\mu}{\sim} z^{d}$. Without loss of generality we may assume that $B=\delta \circ z^{d}$, where $\delta$ is a Möbius transformation, so that $G(B)=\mathcal{D}$ by Lemma 4.1. and

$$
\gamma_{B}(G(B))=\delta \circ \mathcal{D} \circ \delta^{-1} .
$$

By Lemma 4.3. either $G_{0}(B)$ is a group of order at most four, or $\delta \in \mathcal{D}$. In the first case, the number of classes $X$ in $\overline{\varepsilon_{0}(B)}$ with non-ramified $\mathcal{O}_{1}^{X}$ does not exceed four, and inequality (50) still holds. On the other hand, in the second case $B=c z^{ \pm d}, c \in \mathbb{C} \backslash\{0\}$, implying that $B$ is conjugate to $z^{ \pm d}$ in contradiction with the assumption.

The case where $\mathcal{O}=\mathcal{O}_{1}^{X}$ is ramified reduces to the previous one. Indeed, since (48) is a minimal holomorphic map, it follows from Theorem 2.20 that there exists a rational function $F$ such that for any $X$ with $\mathcal{O}_{1}^{X}=\mathcal{O}$ the diagram

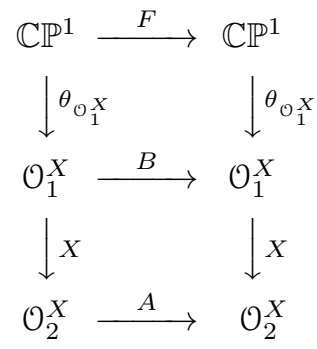

commutes, and the triple $B, \theta_{\mathcal{O}_{1}^{X}}, F$ is a primitive solution of equation (3). Set

$$
X^{\prime}=X \circ \theta_{\mathcal{O}_{1}^{X}}
$$


Since $X^{\prime}=\theta_{\mathcal{O}_{2}^{X}}$ by (16), it follows from the commutativity of diagram (51) by Theorem 2.18 that any $X \in \overline{\mathcal{E}_{0}(B)}$ with $\mathcal{O}_{1}^{X}=\mathcal{O}$ lifts to some $X^{\prime} \in \overline{\mathcal{E}_{0}(F)}$ with non-ramified $\mathcal{O}_{1}^{X^{\prime}}$. Moreover, since $B$ is not a special function, $F$ also is not a special function, by Lemma 2.13. Since $\operatorname{deg} F=\operatorname{deg} B$, this yields that the number of classes $X$ in $\overline{\mathcal{E}_{0}(B)}$ with $\mathcal{O}_{1}^{X}=\mathcal{O}$ can be bounded from above by a number depending on $d$ only. Lastly, the inequality

$$
\operatorname{deg} X \leq \max \{60,2 d\}
$$

still holds since

$$
\operatorname{deg} X<\operatorname{deg} X^{\prime}=\operatorname{deg} \theta_{\mathcal{O}_{2}^{X}}
$$

\section{Description of $\mathcal{E}(B)$ FOR NON-SPECIAL $B$}

In this section, we describe the structure of the set $\mathcal{E}(B)$ for non-special rational functions $B$, and prove Theorem 1.1 and Theorem 1.2. We also prove an effective version of the Ritt theorem.

Theorem 5.1. Let $B$ be a non-special rational function of degree $d \geq 2$. Then any $X \in \mathcal{E}(B)$ can be represented in the form

$$
X=X^{\prime} \circ U \circ B^{\circ k}, \quad k \geq 0,
$$

where $\chi\left(\mathcal{O}_{2}^{X^{\prime}}\right)>0$ and $U$ is a compositional right factor of some iterate $B^{\circ l}, l \geq 0$. Furthermore,

$$
\operatorname{deg} X^{\prime} \leq \max \{60,2 d\}
$$

and

$$
l \leq\left(\sigma_{0}(d)-2\right)\left(12 \log _{2} d+11\right),
$$

where $\sigma_{0}(d)$ is the number of divisors function.

Proof. If $\mathbb{C}(X, B)=\mathbb{C}(z)$, then the statements of the theorem holds for $l=0$ and $k=0$ by Theorem 4.4. So, assume that $\mathbb{C}(X, B) \neq \mathbb{C}(x)$. Without loss of generality we may assume that $X$ is not a rational function in $B^{\circ k}, k \geq 1$, since if $X=\widehat{X} \circ B^{\circ k}$ is contained in $\mathcal{E}(B)$, then $\widehat{X}$ also is contained in $\mathcal{E}(B)$.

We recall that any solution $A, X, B$ of (31) can be reduced to a primitive one as follows. Let $\mathbb{C}(X, B)=\mathbb{C}\left(U_{1}\right)$, where $U_{1} \in \mathbb{C}(z)$, and let $X_{1}, V_{1}$ be rational functions such that

$$
X=X_{1} \circ U_{1}, \quad B=V_{1} \circ U_{1},
$$

and $\mathbb{C}\left(X_{1}, V_{1}\right)=\mathbb{C}(z)$. Substituting expressions (53) in (3) we see that the diagram

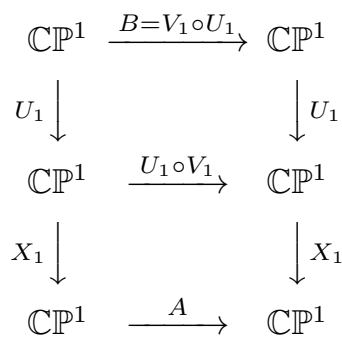

commutes. Thus,

$$
A^{\prime}=A, \quad X^{\prime}=X_{1}, \quad B^{\prime}=U_{1} \circ V_{1}
$$


is also a solution of (3). This new solution is not necessary primitive. Nevertheless, $\operatorname{deg} X_{1}<\operatorname{deg} X$. Therefore, after a finite number of similar transformations we will arrive to a primitive solution.

In more detail, we can find rational functions $X_{i}, V_{i}, U_{i}, 1 \leq i \leq l$, such that

$$
\begin{gathered}
\mathbb{C}\left(X_{i}, U_{i} \circ V_{i}\right)=\mathbb{C}\left(U_{i+1}\right), \quad \operatorname{deg} U_{i+1}>1, \quad 1 \leq i \leq l-1, \\
X_{i}=X_{i+1} \circ U_{i+1}, \quad U_{i} \circ V_{i}=V_{i+1} \circ U_{i+1}, \quad 1 \leq i \leq l-1, \\
\mathbb{C}\left(X_{i}, V_{i}\right)=\mathbb{C}(z), \quad 1 \leq i \leq l,
\end{gathered}
$$

and

$$
\mathbb{C}\left(X_{l}, U_{l} \circ V_{l}\right)=\mathbb{C}(z)
$$

Setting

$$
B_{i}=U_{i} \circ V_{i}=V_{i+1} \circ U_{i+1}, \quad 1 \leq i \leq l-1,
$$

and

$$
U=U_{l} \circ \cdots \circ U_{2} \circ U_{1},
$$

we see that by construction

$$
X=X_{l} \circ U
$$

the diagram

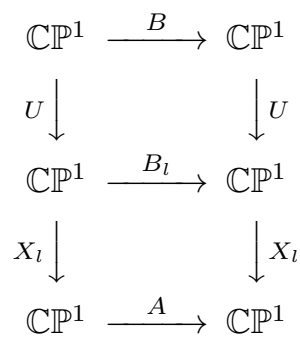

commutes, and $A, X_{l}, B_{l}$ is a primitive solution of (3). Thus, since $U$ is a compositional right factor of $B^{\circ l}$ by Lemma 3.1, and the inequalities $\operatorname{deg} X_{l} \leq \max \{60,2 d\}$ and $\chi\left(\mathcal{O}_{2}^{X_{l}}\right)>0$ hold by Theorem 4.4, in order to prove the theorem we only must show that $l$ satisfies inequality (52).

Since the first equality in (54) implies that any compositional right factor of $U_{i+1}$ is a compositional right factor of $X_{i}$, it follows from (55) that

$$
\mathbb{C}\left(V_{i}, U_{i+1}\right)=\mathbb{C}(z), \quad 1 \leq i \leq l-1 .
$$

In turn, (57) yields that

$$
\operatorname{deg} U_{i+1} \leq \operatorname{deg} U_{i}, \quad i \geq 1 .
$$

Indeed, denote by $I$ the imprimitivity system of the monodromy group of $B_{i}$ corresponding to the decomposition $B_{i}=V_{i+1} \circ U_{i+1}$, and by $J$ the imprimitivity system corresponding to the decomposition $B_{i}=U_{i} \circ V_{i}$. Since each block of $I$ contains $\operatorname{deg} U_{i+1}$ elements, while the number of blocks of $J$ is equal to $\operatorname{deg} U_{i}$, if $\operatorname{deg} U_{i+1}>\operatorname{deg} U_{i}$, then there is a block of $J$ containing at least two elements from a block of $I$, implying that $\mathbb{C}\left(V_{i}, U_{i+1}\right) \neq \mathbb{C}(z)$.

By assumption, $\operatorname{deg} U_{i} \geq 2,1 \leq i \leq l$. On the other hand, since $X$ is not a rational function in $B$ the inequality $\operatorname{deg} U_{1}<d$ holds, implying by (58) that 
$\operatorname{deg} U_{i}<d, 1 \leq i \leq l$. Thus, the functions $V_{i}, U_{i}, 1 \leq i \leq l$, have degree at least two and hence the sequence

$$
U_{i} \circ V_{i}=V_{i+1} \circ U_{i+1}, \quad 1 \leq i \leq l-1,
$$

is a chain. We denote this chain by $\mathcal{C}$. Let

$$
1 \leq k_{1} \leq \cdots \leq k_{r} \leq l
$$

be indices such that

and

$$
\begin{gathered}
\operatorname{deg} U_{1}=\operatorname{deg} U_{2}=\cdots=\operatorname{deg} U_{k_{1}}, \\
\operatorname{deg} U_{k_{1}+1}=\operatorname{deg} U_{k_{1}+2}=\cdots=\operatorname{deg} U_{k_{2}}, \\
\cdots \\
\operatorname{deg} U_{k_{r}+1}=\operatorname{deg} U_{k_{r}+2}=\cdots=\operatorname{deg} U_{l},
\end{gathered}
$$

$$
\operatorname{deg} U_{1}>\operatorname{deg} U_{k_{1}+1}>\operatorname{deg} U_{k_{2}+1}>\cdots>\operatorname{deg} U_{k_{r}+1} .
$$

Setting for convenience $k_{0}=0, k_{r+1}=l$, we see that in view of conditions (57) and (59), for any $j, 0 \leq j \leq r$, the subchain

$$
U_{i} \circ V_{i}=V_{i+1} \circ U_{i+1}, \quad k_{j}+1 \leq i \leq k_{j+1}-1,
$$

of the chain $\mathcal{C}$ is good by Lemma 2.15. Therefore, by Theorem 3.3, its length $k_{j+1}-k_{j}$ is less than or equal to $12 \log _{2} d+11$, unless its basis is special. On the other hand, since by construction

$$
B \rightarrow B_{1} \rightarrow B_{2} \rightarrow \cdots \rightarrow B_{l}
$$

is a chain of elementary transformations, it follows from Lemma 2.11 that the basis of (61) is special if and only if $B$ is special. Thus, we conclude that

$$
l=\sum_{j=0}^{r}\left(k_{j+1}-k_{j}\right) \leq(r+1)\left(12 \log _{2} d+11\right),
$$

unless $B$ is special. Finally, since $\operatorname{deg} U_{i}, 1 \leq i \leq l$, is a proper divisor of $d$, it follows from (60) that that

$$
r+1 \leq \sigma_{0}(d)-2
$$

Proof of Theorem 1.1. Assume that $X$ is an element of $\mathcal{E}(B)$ such that $X$ is not a rational function in $B^{\circ k}, k \geq 1$, and consider diagram (56). Since decompositions of a rational function $R$ of degree $n$ into a composition of rational functions $R=L \circ M$, considered up to the change

$$
L=L \circ \nu^{-1}, \quad M=\nu \circ M,
$$

where $\nu$ is a Möbius transformation, correspond to imprimitivity systems of the monodromy group $\operatorname{Mon}(R) \subset S_{n}$ of $R$, there exists a function $\omega: \mathbb{N} \rightarrow \mathbb{N}$ such that, up to the change (62), any rational function of degree $n$ has at most $\omega(n)$ decompositions into a composition of rational functions. Thus, since $U$ is a compositional right factor of $B^{\circ l}$, where $l$ satisfies (52), there exist $W_{1}, W_{2}, \ldots, W_{s}$, where $s$ can be bounded from above by a number depending on $d$ only, such that $U$ in (56) has the form $U=\nu \circ W_{j}$ for some $j, 1 \leq j \leq s$, and a Möbius transformation $\nu$. Besides, it is clear that

$$
\operatorname{deg} W_{j} \leq \operatorname{deg} B^{\circ l}=d^{l}, \quad 1 \leq j \leq s .
$$


Changing in diagram (56) the function $X_{l}$ to the function $X_{l} \circ \nu^{-1}$, the function $U$ to the function $\nu \circ U$, and the function $B_{l}$ to the function $\nu \circ U \circ \nu^{-1}$, for a convenient Möbius transformation $\nu$, without loss of generality we may assume that $U=W_{j}$, for some $j, 1 \leq j \leq s$. The function $B_{l}$ is defined in a unique way by $U$, and it follows from Theorem 4.4 that for each $B_{l}$, up to the change $X_{l} \rightarrow \mu \circ X_{l}$, where $\mu$ is a Möbius transformation, there exist only finitely many functions $X_{l}$ satisfying (56). Moreover, the number of such functions and their degrees can be bounded from above in terms of $d$ only. Finally, it is clear that if to the function $X_{l}$ corresponds the function $A$, then to the function $\mu \circ X_{l}$ corresponds the conjugate function $\mu \circ A \circ \mu^{-1}$.

Remark 5.2. Since $A \sim B$ implies that $B$ is semiconjugate to $A$, Theorem 1.1yields in particular that if $B$ is not special, then $[B]$ contains only finitely many conjugacy classes. This result also follows from the McMullen theorem [10] about isospectral rational functions (see [16]). However, the McMullen theorem is non-effective, while Theorem 1.1 asserts that the number of classes in $[B]$ is bounded from above by a number depending on $\operatorname{deg} B$ only.

Proof of Theorem 1.2. Assume that $X$ commutes with $B$. Without loss of generality we may assume that $X$ is not a rational function in $B^{\circ k}, k \geq 1$, since if

$$
X=\widehat{X} \circ B^{\circ k}
$$

commutes with $B$, then $\widehat{X}$ also commutes with $B$. By Theorem 1.1, there exist $X_{1}, X_{2}, \ldots, X_{r} \in \mathcal{E}(B)$ such that $X=\mu \circ X_{j}$ for some $j, 1 \leq j \leq r$, and a Möbius transformation $\mu$. Therefore, in order to prove Theorem 1.2, it is enough to show that for any $X \in \mathcal{E}(B)$ there exist only finitely many $\mu$ such that $\mu \circ X$ commutes with $B$.

Let us take arbitrary $\mu_{0}$ such $\mu_{0} \circ X$ commutes with $B$, and assume that $\mu \circ X$ also commutes with $B$. We have:

$$
B \circ \mu_{0} \circ X=\mu_{0} \circ X \circ B=\left(\mu_{0} \circ \mu^{-1}\right) \circ \mu \circ X \circ B=\left(\mu_{0} \circ \mu^{-1}\right) \circ B \circ \mu \circ X .
$$

Therefore,

$$
B \circ \mu_{0}=\left(\mu_{0} \circ \mu^{-1}\right) \circ B \circ \mu,
$$

implying that $\nu=\mu_{0} \circ \mu^{-1}$ commutes with $B$. Since the number of Möbius transformation commuting with $B$ is finite, this implies the required statement.

In conclusion, we prove the following effective version of the Ritt theorem (cf. [23], 3]).

Theorem 5.3. Let $B$ and $X$ be commuting rational functions of degree at least two. If $B$ is not special, then $X^{\circ l}=B^{\circ k}$ for some $l, k \geq 1$. Furthermore, there exists a (computable) function $\delta: \mathbb{N} \rightarrow \mathbb{N}$ such that for any non-special $B$ of degree $d$ the number $l$ is bounded from above by $\delta(d)$. On the other hand, if $B$ is special and $\mathcal{O}$ is an orbifold such that $B: \mathcal{O} \rightarrow \mathcal{O}$ is a covering map between orbifolds, then $X: \mathcal{O} \rightarrow \mathcal{O}$ is also a covering map between orbifolds.

Proof. The first part of the theorem follows from Theorem 1.2 (see the introduction). So, assume that $B$ is special, and let $\mathcal{O}=\left(\mathbb{C P}^{1} \backslash \mathcal{E}_{B}, \nu\right)$ be an orbifold such that $B: \mathcal{O} \rightarrow \mathcal{O}$ is a covering map. We observe first that

$$
X^{-1}\left(\mathcal{E}_{B}\right)=\mathcal{E}_{B}
$$


Indeed, since $B^{-1}\left(\mathcal{E}_{B}\right)=\mathcal{E}_{B}$, it follows from the commutativity of the diagram

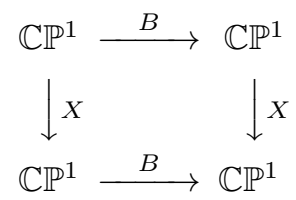

that

$$
B^{-1}\left(X^{-1}\left(\mathcal{E}_{B}\right)\right)=X^{-1}\left(\mathcal{E}_{B}\right) .
$$

Therefore, $X^{-1}\left(\mathcal{E}_{B}\right) \subseteq \mathcal{E}_{B}$. Since the set $X^{-1}\left(\mathcal{E}_{B}\right)$ contains at least $\left|\mathcal{E}_{B}\right|$ points, this implies 663).

Set $R=\mathbb{C P}^{1} \backslash \mathcal{E}_{B}$ and

$$
F=B \circ X=X \circ B .
$$

Since $X: R \rightarrow R$ and $F: R \rightarrow R$ are branched covering maps by (63), we can define the orbifolds $X^{*} \mathcal{O}$ and $F^{*} \mathcal{O}$. By Theorem 2.3, we have:

$$
F^{*} \mathcal{O}=X^{*}\left(B^{*} \mathcal{O}\right) \text {. }
$$

However, since $B: \mathcal{O} \rightarrow \mathcal{O}$ is a covering map, the equality $B^{*} \mathcal{O}=\mathcal{O}$ holds, implying that $F^{*} \mathcal{O}=X^{*} \mathcal{O}$. Thus, $F: X^{*} \mathcal{O} \rightarrow \mathcal{O}$ is a minimal holomorphic map between orbifolds. It follows now from $F=X \circ B$ by Corollary 2.5 that $B: X^{*} \mathcal{O} \rightarrow X^{*} \mathcal{O}$ also is a minimal holomorphic map. In particular, $\chi\left(X^{*} \mathcal{O}\right) \geq 0$ by (12). On the other hand, since $X: X^{*} \mathcal{O} \rightarrow \mathcal{O}$ is a minimal holomorphic map, it follows from (12) that $\chi\left(X^{*} \mathcal{O}\right) \leq 0$. Therefore,

$$
\chi\left(X^{*} \mathcal{O}\right)=0,
$$

implying by Proposition 2.2 that $B: X^{*} \mathcal{O} \rightarrow X^{*} \mathcal{O}$ and $X: X^{*} \mathcal{O} \rightarrow \mathcal{O}$ are covering maps.

Since $X: X^{*} \mathcal{O} \rightarrow \mathcal{O}$ is a covering map, if $\mathcal{E}_{B}$ consists of two points, then (64) implies that the both orbifolds $\mathcal{O}$ and $X^{*} \mathcal{O}$ are non-ramified, so that

$$
X^{*} \mathcal{O}=\mathcal{O} .
$$

Thus, $X: \mathcal{O} \rightarrow \mathcal{O}$ is a covering map, as required. On the other hand, if $\mathcal{E}_{B}$ consists of one point, then without loss of generality we may assume that $\nu(-1)=\nu(1)=2$ and $B= \pm T_{d}$, while (64) implies that the orbifold $X^{*} \mathcal{O}$ is defined by the equality $\widehat{\nu}(a)=\widehat{\nu}(b)=2$, where $a$ and $b$ are some points on $\mathbb{C P}^{1}$. It is not hard to see however that $\pm T_{d}: X^{*} \mathcal{O} \rightarrow X^{*} \mathcal{O}$ cannot be a covering map for such $X^{*} \mathcal{O}$ unless $\{a, b\}=\{-1,1\}$. Thus, in this case equality (65) is also satisfied.

Finally, if $\varepsilon_{B}=\emptyset$, then $B$ is a Lattés map, and it is well-known that the orbifold $\mathcal{O}$ such that $B: \mathcal{O} \rightarrow \mathcal{O}$ is a covering map is defined in a unique way by the dynamics of $B$ (see [12], or [20, Theorem 6.1]). Thus, equality (65) still holds.

\section{REFERENCES}

[1] Xavier Buff and Adam L. Epstein, From local to global analytic conjugacies, Ergodic Theory Dynam. Systems 27 (2007), no. 4, 1073-1094, DOI 10.1017/S0143385707000041. MR.2342966

[2] Charles W. Curtis and Irving Reiner, Representation theory of finite groups and associative algebras, Pure and Applied Mathematics, Vol. XI, Interscience Publishers, a division of John Wiley \& Sons, New York-London, 1962. MR.0144979

[3] A. È. Erëmenko, Some functional equations connected with the iteration of rational functions (Russian), Algebra i Analiz 1 (1989), no. 4, 102-116; English transl., Leningrad Math. J. 1 (1990), no. 4, 905-919. MR.1027462 
[4] Alexandre Eremenko, Invariant curves and semiconjugacies of rational functions, Fund. Math. 219 (2012), no. 3, 263-270, DOI 10.4064/fm219-3-5. MR3001243

[5] H. M. Farkas and I. Kra, Riemann surfaces, 2nd ed., Graduate Texts in Mathematics, vol. 71, Springer-Verlag, New York, 1992. MR1139765

[6] P. Fatou, Sur l'iteration analytique et les substitutions permutables, J. Math. Pures Appl. 2 (1923), no. 9, 343-384.

[7] Hiroyuki Inou, Extending local analytic conjugacies, Trans. Amer. Math. Soc. 363 (2011), no. 1, 331-343, DOI 10.1090/S0002-9947-2010-05049-4. MR2719684

[8] Gaston Julia, Mémoire sur la permutabilité des fractions rationnelles (French), Ann. Sci. École Norm. Sup. (3) 39 (1922), 131-215. MR.1509242

[9] Felix Klein, Lectures on the icosahedron and the solution of equations of the fifth degree, Second and revised edition, Dover Publications, Inc., New York, N.Y., 1956. Translated into English by George Gavin Morrice. MR0080930

[10] Curt McMullen, Families of rational maps and iterative root-finding algorithms, Ann. of Math. (2) 125 (1987), no. 3, 467-493, DOI 10.2307/1971408. MR890160

[11] Alice Medvedev and Thomas Scanlon, Invariant varieties for polynomial dynamical systems, Ann. of Math. (2) 179 (2014), no. 1, 81-177, DOI 10.4007/annals.2014.179.1.2. MR3126567

[12] John Milnor, On Lattès maps, Dynamics on the Riemann sphere, Eur. Math. Soc., Zürich, 2006, pp. 9-43, DOI 10.4171/011-1/1. MR2348953

[13] John Milnor, Dynamics in one complex variable, 3rd ed., Annals of Mathematics Studies, vol. 160, Princeton University Press, Princeton, NJ, 2006. MR2193309

[14] Fedor Pakovich, On semiconjugate rational functions, Geom. Funct. Anal. 26 (2016), no. 4, 1217-1243, DOI 10.1007/s00039-016-0383-6. MR3558309

[15] Fedor Pakovich, On algebraic curves $A(x)-B(y)=0$ of genus zero, Math. Z. 288 (2018), no. 1-2, 299-310, DOI 10.1007/s00209-017-1889-9. MR3774414

[16] Fedor Pakovich, Recomposing rational functions, Int. Math. Res. Not. IMRN 7 (2019), 19211935, DOI 10.1093/imrn/rnx172. MR3938311

[17] Fedor Pakovich, Polynomial semiconjugacies, decompositions of iterations, and invariant curves, Ann. Sc. Norm. Super. Pisa Cl. Sci. (5) 17 (2017), no. 4, 1417-1446. MR3752532

[18] Fedor Pakovich, On rational functions whose normalization has genus zero or one, Acta Arith. 182 (2018), no. 1, 73-100, DOI 10.4064/aa170113-28-8. MR3740243

[19] F. Pakovich, Semiconjugate rational functions: a dynamical approach, Arnold Math. J. 4 (2018), no. 1, 59-68, DOI 10.1007/s40598-018-0081-6. MR3810568

[20] F. Pakovich, On generalized Lattès maps, J. Anal. Math., accepted.

[21] F. Pakovich, Algebraic curves $A^{\circ l}(x)-U(y)=0$ and arithmetic of orbits of rational functions, Mosc. Math. J. 20 (2020), no. 1, 153-183, DOI 10.17323/1609-4514-2020-20-1-153-183. MR 4060316

[22] J. F. Ritt, On the iteration of rational functions, Trans. Amer. Math. Soc. 21 (1920), no. 3, 348-356, DOI 10.2307/1988936. MR1501149

[23] J. F. Ritt, Permutable rational functions, Trans. Amer. Math. Soc. 25 (1923), no. 3, 399-448, DOI 10.2307/1989297. MR1501252

Department of Mathematics, Ben Gurion University of the Negev, P.O.B. 653, Beer SHEVA, 8410501 ISRAEL

Email address: pakovich@math.bgu.ac.il 